\title{
The impact of AGN wind feedback in simulations of isolated galaxies with a multiphase ISM
}

\author{
Paul Torrey ${ }^{\oplus},{ }^{1 \star}$ Philip F. Hopkins ${ }^{\oplus},{ }^{2}$ Claude-André Faucher-Giguère ${ }^{\oplus, 3}$ Daniel Anglés-Alcázar, ${ }^{4,5}$ \\ Eliot Quataert ${ }^{\oplus},{ }^{6}$ Xiangcheng Ma ${ }^{\oplus},{ }^{6}$ Robert Feldmann ${ }^{\oplus}, 7$ Dusan Keres ${ }^{8}$ and Norm Murray ${ }^{9} \dagger$ \\ ${ }^{1}$ Department of Astronomy, University of Florida, 211 Bryant Space Sciences Center, Gainesville, FL 32611, USA \\ ${ }^{2}$ TAPIR, California Institute of Technology, Mailcode 350-17, Pasadena, CA 91125, USA \\ ${ }^{3}$ Department of Physics and Astronomy and CIERA, Northwestern University, 2145 Sheridan Road, Evanston, IL 60208, USA \\ ${ }^{4}$ Center for Computational Astrophysics, Flatiron Institute, 162 Fifth Avenue, New York, NY 10010, USA \\ ${ }^{5}$ Department of Physics, University of Connecticut, 196 Auditorium Road, U-3046, Storrs, CT 06269-3046, USA \\ ${ }^{6}$ Department of Astronomy and Theoretical Astrophysics Center, University of California Berkeley, Berkeley, CA 94720, USA \\ ${ }^{7}$ Institute for Computational Science, University of Zurich, CH-8057 Zurich, Switzerland \\ ${ }^{8}$ Department of Physics, Center for Astrophysics and Space Sciences, University of California at San Diego, 9500 Gilman Drive, La Jolla, CA 92093, USA \\ ${ }^{9}$ Canadian Institute for Theoretical Astrophysics, 60 St George Street, University of Toronto, Toronto, ON M5S 3H8, Canada
}

Accepted 2020 July 20. in original form 2020 June 24

\begin{abstract}
Accreting black holes can drive fast and energetic nuclear winds that may be an important feedback mechanism associated with active galactic nuclei (AGN). In this paper, we implement a scheme for capturing feedback from these fast nuclear winds and examine their impact in simulations of isolated disc galaxies. Stellar feedback is modelled using the Feedback In Realistic Environments (FIRE) physics and produces a realistic multiphase interstellar medium (ISM). We find that AGN winds drive the formation of a low-density, high-temperature central gas cavity that is broadly consistent with analytic model expectations. The effects of AGN feedback on the host galaxy are a strong function of the wind kinetic power and momentum. Low- and moderate-luminosity AGN do not have a significant effect on their host galaxy: the AGN winds inefficiently couple to the ambient ISM and instead a significant fraction of their energy vents in the polar direction. For such massive black holes, accretion near the Eddington limit can have a dramatic impact on the host galaxy ISM: if AGN wind feedback acts for $\gtrsim 20-30$ Myr, the inner $\sim 1-10 \mathrm{kpc}$ of the ISM is disrupted and the global galaxy star formation rate is significantly reduced. We quantify the properties of the resulting galaxy-scale outflows and find that the radial momentum in the outflow is boosted by a factor of $\sim 2-3$ relative to that initially supplied in the AGN wind for strong feedback scenarios, decreasing below unity for less energetic winds. In contrast to observations, however, the outflows are primarily hot, with very little atomic or molecular gas. We conjecture that merging galaxies and high-redshift galaxies, which have more turbulent and thicker discs and very different nuclear gas geometries, may be even more disrupted by AGN winds than found in our simulations.
\end{abstract}

Key words: galaxies: evolution-galaxies: ISM-galaxies: nuclei-quasars: supermassive black holes.

\section{INTRODUCTION}

Feedback from supermassive black holes - commonly called active galactic nuclei (AGN) feedback - has been postulated as an important component of galaxy formation. While it is broadly accepted that supermassive black holes are present at the centre of all massive galaxies (e.g. Ho 2008) and that they can release sufficiently large amounts of energy during accretion events to remove all gas from a galaxy (e.g. Ciotti \& Ostriker 1997; Silk \& Rees 1998; King 2003; Wyithe \& Loeb 2003; Murray, Quataert \& Thompson 2005), the actual significance of AGN feedback on the formation history of their host galaxy remains an unsettled and debated topic (see Kormendy \& Ho 2013, and references within). Much of the uncertainty regarding

^E-mail: paul.torrey@ufl.edu

$\dagger$ Canada Research Chair in Astrophysics. the impact of AGN feedback stems from a lack of a clear and physical picture for how AGN couple their energy to the ambient interstellar medium (ISM) and/or circumgalactic medium (CGM). Despite the very large feedback energy budget associated with AGN, it is as yet not known how AGN allocate their energy budget between e.g. radiation, relativistic jets, subrelativistic winds, etc., nor how efficiently these varied feedback mechanisms couple to the host galaxy's ISM (e.g. Wagner, Bicknell \& Umemura 2012).

Perhaps the best empirical motivation that galaxies regulate their growth through AGN feedback is the existence of tight correlations between supermassive black hole mass and host galaxy properties such as bulge mass and velocity dispersion (Ferrarese \& Merritt 2000; Gebhardt et al. 2000). These black hole/host galaxy scaling relations can be explained if black hole feedback regulates its own growth and perhaps the stellar mass growth of the host galaxy. In addition to the scaling relations, observations of bright quasars show outflowing 
molecular gas (e.g. Sturm et al. 2011; Cicone et al. 2014; Tombesi et al. 2015; Kakkad et al. 2017), neutral and ionized atomic gas (e.g. Moe et al. 2009; Rupke \& Veilleux 2011; Liu et al. 2013; Harrison et al. 2014; Carniani et al. 2015, 2016), and more highly ionized gas probed by X-ray absorption or emission (e.g. Cappi et al. 2009; Tombesi et al. 2010; Greene et al. 2014; Veilleux et al. 2014). These outflows often reach velocities in excess of $v>1000 \mathrm{~km} \mathrm{~s}^{-1}$ (e.g. Nesvadba et al. 2006, 2008; Sturm et al. 2011; Zakamska et al. 2016; Williams et al. 2017) and can have mass outflow rates $\dot{M}_{\text {out }}>$ $1000 \mathrm{M}_{\odot} \mathrm{yr}^{-1}$ (e.g. Maiolino et al. 2012). The observed properties of these outflows provide observational guidance into how AGN may be able to self-regulate their own growth, modulate the stellar mass growth of the host galaxy, and establish black hole/host galaxy scaling relations (e.g. Wylezalek \& Zakamska 2016).

However, it is not clear that all AGN have a strong - or even significant - impact on galaxy scales. Indeed, most AGN have relatively low luminosity and may simply not be energetic enough to impact galactic scales (e.g. Hopkins \& Hernquist 2006; Heckman \& Best 2014). In particular, the majority of low-redshift AGN are weak, low-level Seyferts hosted by galaxies that show little sign of being impacted by the central AGN (e.g. Ho 2008; Kim, Ostriker \& Kim 2013). This has led to multiple claims that AGN do not significantly impact galaxies, and therefore may not be critical to galaxy evolution. We note, though, that compilations of AGN outflow observations suggest that only relatively luminous $\mathrm{AGN}\left(L_{\mathrm{AGN}} \gtrsim 10^{45} \mathrm{erg} \mathrm{s}^{-1}\right)$ drive powerful winds on galaxy scales (e.g. Rupke \& Veilleux 2013; Cicone et al. 2014; Fiore et al. 2017; Fluetsch et al. 2019), so that a meaningful discussion of AGN feedback must distinguish between different luminosity regimes.

On the theoretical side, it has been shown that relationships between black hole mass and galaxy properties similar to observed scaling relations can be produced in models without AGN feedback, either because of the black hole fuelling physics (e.g. Anglés-Alcázar, Özel \& Davé 2013; Anglés-Alcázar et al. 2015, 2017a,c) or as a result of the central limit theorem associated with galaxy mergers (e.g. Peng 2007; Jahnke \& Macciò 2011). It is important to note, though, that this does not imply that AGN feedback plays no role in explaining observed scaling relations or galaxy properties.

In this paper, we focus on one main mode of AGN feedback: the coupling of fast, nuclear kinetic winds to the host galaxy ISM. Simulations of accretion discs around black holes indicate that rapidly accreting black holes can launch nuclear winds via radiation pressure on atomic lines (e.g. Proga, Stone \& Kallman 2000). Observationally, these winds are detected as broad absorption lines (BALs) in the rest-ultraviolet (UV) or ultrafast outflows (UFOs) in X-rays, and have velocities ranging from $\sim 5000 \mathrm{~km} \mathrm{~s}^{-1}$ to $>0.3 c$ (Weymann, Carswell \& Smith 1981; Tombesi et al. 2010; Nardini et al. 2015). The energy associated with such a fast wind can be significant, despite the relatively modest mass outflow rates on small scales. The energetics of these nuclear winds can also be constrained by observations of the ambient gas that they sweep up when expanding into host galaxies (Faucher-Giguère, Quataert \& Murray 2012; Stern et al. 2016). When such a wind runs into an ambient ISM, it is expected to shock to very high temperatures and create an overpressurized central gas bubble (e.g. King 2003; Faucher-Giguère \& Quataert 2012; Richings \& Faucher-Giguère 2018a,b). This overpressurized gas bubble can do ' $P \mathrm{~d} V$ ' work on the surrounding ISM, potentially leading to a large radial momentum enhancement. The magnitude of the radial momentum boost and its effects on galaxies depend critically on how effectively the hot shocked gas is confined by the ambient medium, as opposed to the wind energy efficiently venting out of the galaxy (e.g. Gabor \&
Bournaud 2014). This, in turn, depends sensitively on the geometry and dynamics of the multiphase ISM. Simulations are therefore needed to resolve the theoretical uncertainties involved.

In this work, we use idealized simulations of isolated galaxies to investigate this problem. Although we do not include the full cosmological context, our simulations explicitly treat the multiphase ISM self-consistently shaped by gravity, cooling, and stellar feedback. Specifically, we employ the GIZMO simulation code including the FIRE feedback model (Hopkins et al. 2014, 2018b) in conjunction with a new 'particle spawning' method for simulating AGN-driven winds. Our simulations include a kinetic model for BAL winds and Compton heating/cooling off the associated AGN radiation. As our simulations include entire, porous galactic discs, they allow us to analyse how black hole feedback acts on realistic disc galaxies. This effort builds upon a number of previous studies aiming to understand the coupling of AGN feedback to the gas in galaxies, including the impact of gas geometry and gas porosity (e.g. Choi et al. 2012; Wagner, Umemura \& Bicknell 2013; Costa, Sijacki \& Haehnelt 2014; Curtis \& Sijacki 2016; Zubovas \& Bourne 2017; Costa et al. 2018; Zubovas 2018).

The structure of this paper is as follows. In Section 2, we outline our methods, including a brief description of the GIZMO simulation code, the FIRE stellar physics, the new methods employed in this paper for simulating BAL winds, and the initial conditions employed in the paper. In Section 3, we characterize the impact of BAL winds on properties of this host galaxy, specifically focusing on the emergence of a hot, low-density central cavity, and any impact of galaxy cold gas content or star formation rates (SFRs). In Section 4, we discuss the properties of the outflows that are driven by the fast winds, including a description of the velocity and phase distributions. We discuss our results in Section 5 and conclude in Section 6.

\section{METHODS}

We present a new simulation suite to probe disc galaxies under the influence of stellar feedback and AGN-driven winds. The simulation code - including the employed AGN feedback models - and initial conditions used in this paper are presented in this section.

\subsection{Simulation code}

We use the $N$-body/hydrodynamic simulation code GIZMO (Hopkins 2015) to carry out the simulations presented in this paper. GIZMO includes a comprehensive set of physical prescriptions used for galaxy formation models (Hopkins et al. 2014, 2018b). Throughout this paper we employ GIZMO using the meshless finite mass (MFM) scheme to solve the hydrodynamic equations.

In addition to gravity and hydrodynamics, GIZMO is able to model a number of the physical processes that are of central importance to galaxy formation. In particular, GIZMO is equipped to handle radiative cooling of gas down to $\sim 10 \mathrm{~K}$, star formation, and feedback from young stars including supernova, stellar winds, radiation pressure, and photoionization. The galaxy formation physics model employed in this paper has been described in detail in Hopkins et al. (2018b), where we refer readers who are interested in the cooling physics, star formation, or stellar feedback implementations used in our simulations. Briefly, radiative cooling is followed including a variety of processes, accounting for local and metagalactic radiation sources in multiple bands and 11 explicitly evolved metal species; star formation occurs in gas that is locally self-gravitating, Jeansunstable, self-shielded, and above a density threshold $>1000 \mathrm{~cm}^{-3}$; once formed stars act on their environment via a variety of processes 
including mechanical feedback from SNe Types Ia and II and O/B and AGB mass loss (Hopkins et al. 2018a), photoelectric and photoionization heating, and single- and multiple-scattering radiation pressure (Hopkins et al. 2020). The FIRE model has been used extensively in idealized galaxy simulations and cosmological simulations to study, among other topics, the role of feedback in shaping the internal properties of galaxies (e.g. El-Badry et al. 2016; Ma et al. 2016, 2017), the impact of stellar feedback on the underlying dark matter halo properties (e.g. Chan et al. 2015; Oñorbe et al. 2015), the properties of the CGM (Faucher-Giguère et al. 2015, 2016; Hafen et al. 2017), the feedback-driven galactic baryon cycle (e.g. Muratov et al. 2015, 2017; Anglés-Alcázar et al. 2017b), and the growth rates of black holes (Anglés-Alcázar et al. 2017a). The physical model employed in this paper is the same as was considered in Torrey et al. (2017), with the exception that the simulations presented in this paper contain a form of AGN feedback as described in this section.

\subsection{Treatment of black holes}

All simulations considered in this paper include supermassive black hole particles that are initialized to be in the centre of each galaxy. The black holes are allowed to move freely (i.e. under the influence of gravity only without any added dynamical friction), but in practice they remain near the centre of the galaxy owing to a combination of the high mass resolution used in these simulations and the idealized nature of the initial conditions.

\subsubsection{Black hole accretion rates}

The black hole accretion rate in all of simulations used in this paper is manually set to the Eddington accretion rate. Specifically, the black hole accretion rates are set to

$\dot{M}_{\mathrm{BH}}=f_{\mathrm{Edd}} \dot{M}_{\mathrm{edd}}=2 f_{\mathrm{Edd}}\left[\frac{\mathrm{M}_{\odot}}{\mathrm{yr}}\right]\left(\frac{M_{\mathrm{BH}}}{10^{8} \mathrm{M}_{\odot}}\right)$,

corresponding to an AGN luminosity of

$L_{\mathrm{BH}}=f_{\mathrm{Edd}} L_{\mathrm{Edd}}=1.3 \times 10^{46} f_{\mathrm{Edd}}\left[\frac{\mathrm{erg}}{\mathrm{s}}\right]\left(\frac{M_{\mathrm{BH}}}{10^{8} \mathrm{M}_{\odot}}\right)$,

where $f_{\mathrm{Edd}}=1$ for all simulations here, $M_{\mathrm{BH}}$ is the black hole mass, and where we have assumed a radiative efficiency of $\epsilon_{\mathrm{r}}=$ 0.1 . Manually fixing the black hole accretion rate can, in principle, lead to periods of time where the black hole continues to experience a high accretion rate - and inject accordingly strong feedback - even though the local gas density has been significantly reduced. The present set-up with a fixed and prescribed accretion rate is designed to enable a clean and controlled numerical experiment concerning the interaction of time-steady nuclear outflows with disc galaxies. If we allowed the black hole accretion rates to adjust based on the local gas density, some of the detailed conclusions regarding feedback strength may be modified. Even though the accretion rate for feedback purposes is fixed, the black hole particles are allowed to accrete simulation particles that are gravitationally bound to the black hole. Particles within the smoothing kernel with velocities less than the escape velocity are fully swallowed by the black hole. Material that is accreted by this method increases the gravitational mass of the black hole particle (i.e. for the gravity calculation), but does not impact the black hole mass used to calculate the accretion rate. In practice, including this accretion prescription has negligible impact on the presented simulations, but will enable future studies with self-consistently determined accretion rates.

\subsubsection{Fast nuclear AGN winds}

The primary AGN/BH feedback process that we consider in this paper represents the category of 'fast' nuclear winds (driven from e.g. the AGN accretion disc, broad-line region, or any other unresolved scale around the $\mathrm{BH}$ ). We refer to these as BAL winds, but emphasize that our model is purely a phenomenological description of any nonrelativistic magnetohydrodynamic (MHD) or hydrodynamic-type, wide-angle outflow that emerges from the central $\lesssim$ pc scales around a BH.

In our BAL wind model, a fraction of the total AGN luminosity is assumed to drive a fast, isotropic wind radially outward from the central supermassive black hole. We assume the black hole has a luminosity of

$L=\epsilon_{\mathrm{r}} \dot{M}_{\mathrm{BH}} c^{2}$,

where $\epsilon_{\mathrm{r}} \approx 0.1$ is the black hole radiative efficiency. Although fast winds expelled from real black holes may have a preferred outflow direction relative to the orientation of the black hole's accretion disc (e.g. Moe et al. 2009; Dunn et al. 2010; Sa̧dowski et al. 2013; Jiang, Stone \& Davis 2014), we adopt an isotropic wind distribution because the actual accretion disc geometry is far below the resolution limit of our simulations, and because the observed winds of interest appear to be relatively wide angle (i.e. not extremely narrowly collimated, as relativistic jets), so the detailed opening angle has relatively small effects.

The wind physical properties in our model are set with two parameters: (i) the relative fraction of gas that is accreted on to the black hole versus driven into the wind, $f_{\mathrm{acc}}$; and (ii) the wind velocity, $v_{\mathrm{BAL}}$. The wind mass launching rate is related to the black hole accretion rate according to $\dot{M}_{\mathrm{BAL}}=\left[\left(1-f_{\mathrm{acc}}\right) / f_{\mathrm{acc}}\right] \dot{M}_{\mathrm{BH}}=\eta_{\mathrm{BAL}} \dot{M}_{\mathrm{BH}}$, where $f_{\text {acc }}$ is the fraction of material that is accreted (versus launched into a wind) and $\eta_{\mathrm{BAL}}$ is the BAL wind mass loading (Ostriker et al. 2010; Choi et al. 2018). The fast wind momentum and energy injection rates are

$\dot{p}_{\mathrm{BAL}}=\eta_{\mathrm{BAL}} \dot{M}_{\mathrm{BH}} v_{\mathrm{BAL}}$

and

$\dot{E}_{\mathrm{BAL}}=\frac{1}{2} \eta_{\mathrm{BAL}} \dot{M}_{\mathrm{BH}} v_{\mathrm{BAL}}^{2}$

Convenient 'units' for the momentum and energy injection rates are $L / c$ and $L$, respectively (and these represent the momentum and energy 'budgets' if the winds are singly scattered via radiation pressure or pure radiative heating, respectively). So it is useful to define the fast wind momentum loading and energy loading as

$\eta_{p}=\frac{\dot{p}_{\mathrm{BAL}}}{L / c}=\frac{\eta_{\mathrm{BAL}} v_{\mathrm{BAL}}}{\epsilon_{\mathrm{r}} c}$

and

$\eta_{E}=\frac{\dot{E}_{\mathrm{BAL}}}{L}=\frac{\eta_{\mathrm{BAL}} v_{\mathrm{BAL}}^{2}}{2 \epsilon_{\mathrm{r}} c^{2}}$.

For our fiducial choices of wind mass loading $\eta_{\mathrm{BAL}}=1$ and $\mathrm{BAL}$ wind speed $v_{\mathrm{BAL}}=0.1 \mathrm{c}=30000 \mathrm{~km} \mathrm{~s}^{-1}$, the BAL wind momentum loading is $\eta_{p}=1$ and the BAL wind energy loading is $\eta_{E}=$ 0.05 . These fiducial values are convenient because (i) they are well motivated by accretion disc simulations (e.g. Proga et al. 2000; Risaliti \& Elvis 2010; Nomura et al. 2013; Sa̧dowski et al. 2013; Jiang et al. 2014; Nomura \& Ohsuga 2017), and (ii) they lead to momentum loading and energy loading values that are consistent with the momentum loading and thermal energy coupling values used in a wide range of previous black hole feedback studies (e.g. 
Di Matteo, Springel \& Hernquist 2005; Sijacki et al. 2007, 2015; Teyssier et al. 2011; Anglés-Alcázar et al. 2017a; Tremmel et al. 2017).

We stress that in so far as the effects of AGN in our simulations are dominated by mechanical feedback, the $\mathrm{BH}$ masses and luminosities (or Eddington ratios) and corresponding factors $\eta_{p}, \eta_{E}$ are all degenerate and essentially exist only as physical motivation. What actually matters is the wind momentum and energy flux input into the simulation $\left(\dot{P}_{\mathrm{BAL}}, \dot{E}_{\mathrm{BAL}}\right)$; we could always attribute the same values of energy/momentum flux to e.g. a more luminous $\mathrm{BH}$ with a lower feedback efficiency (or to a more massive $\mathrm{BH}$ with lower Eddington ratio).

The wind is realized in our simulations by spawning new gas particles in close proximity to the black hole. We spawn new particles - rather than returning mass, momentum, and energy to existing nearby cells - for three specific reasons. First, spawning new particles allows us to impart manually controlled high gas velocities to the wind, which in turn allows us to explicitly resolve the wind shock. Second, while the gas density immediately around the black hole can become quite low (which would force 'neighbourbased' feedback schemes to potentially inject mass, momentum, and energy to nearest neighbours at large distances), the applied particle spawning technique always injects feedback locally around the black hole. Third, in contrast with a neighbour-based feedback coupling scheme, spawning new particles and allowing for self-consistent hydrodynamic coupling naturally captures any impact from the local gas geometry. The total wind mass each black hole particle should return is calculated at each time step according to $M_{\mathrm{w}}=\dot{M}_{\mathrm{w}} \Delta t_{\mathrm{BH}}$, where $\Delta t_{\mathrm{BH}}$ is the current black hole particle's simulation time step. The wind itself is spawned with (several) new gas particles, each with mass approximately equal to a specified target wind mass, $m_{\mathrm{w}, \text { target }}$. The number of wind particles to be spawned at any time step is taken to be the closest rounded integer to $n_{\mathrm{w}}=M_{\mathrm{w}} / m_{\mathrm{w} \text {, target }}$. In the case of $0<n_{\mathrm{w}}<1$, we assign $n_{\mathrm{w}}=1$. We note, however, that it makes little difference if we instead stochastically sampled wind spawning in this regime. In all cases, the actual mass of the newly spawned particles is set by $m_{\mathrm{w}}^{\prime}=M_{\mathrm{w}} / n_{\mathrm{w}}$. Our simulations use typical target wind masses of $\sim 100 \mathrm{M}_{\odot}$, but we have tested variations in this target mass by factors of several and found limited impact on our results. The BAL wind mass originates from an unresolved accretion disc, which is part of the black hole particle's mass. In principle, therefore, the newly spawned wind mass should be subtracted from the total black hole particle's mass. However, since we manually prescribe accretion rates in this paper, we neglect this point, and hold the black hole particle's mass constant while spawning the BAL wind. This represents a source of mass non-conservation in our simulations, but is negligibly important even when integrated over the full simulation time span.

All newly spawned wind particles are placed slightly offset from the black holes location and given radially outward velocities. The exact spawning location is randomly selected such that wind particle spawning locations - and radially outward velocity vectors - uniformly sample the unit sphere. The radial offset from the black hole is $\Delta r=0.1 \mathrm{pc}$ for all simulations in this paper. Each new BAL wind particle is given a temperature of $T=10^{4} \mathrm{~K}$, a metallicity of $Z=Z_{\odot}$, and a velocity vector that has magnitude $v_{\mathrm{BAL}}$. The adopted input wind temperature is consistent with observed BAL winds that have temperatures of $10^{4}<T<10^{5} \mathrm{~K}$. However, we note that the initial wind temperature has little impact on our results since the wind particles quickly heat through shocks as they begin propagating out of the central region. Changing the initial particle temperature by 1-2 orders of magnitude has no impact on our conclusions, as the
Table 1. BAL wind feedback run names and parameters.

\begin{tabular}{lccccc}
\hline Run name & $\begin{array}{c}v_{\mathrm{BAL}}{ }^{a} \\
\left(\mathrm{~km} \mathrm{~s}^{-1}\right)\end{array}$ & $\eta_{\mathrm{BAL}}{ }^{b}$ & $\epsilon_{\mathrm{r}}{ }^{c}$ & $\eta_{p}{ }^{d}$ & $\eta_{E}{ }^{e}$ \\
\hline No feedback & - & 0 & 0 & 0 & 0 \\
Weak & 3000 & 1 & 0.1 & 0.1 & 0.0005 \\
Intermediate & 10000 & 1 & 0.1 & 0.333 & 0.005 \\
Strong & 30000 & 1 & 0.1 & 1 & 0.05
\end{tabular}

${ }^{a}$ BAL wind velocity.

${ }^{b} \mathrm{BAL}$ wind mass loading defined such that $\eta_{\mathrm{BAL}}=\dot{M}_{\mathrm{BAL}} / \dot{M}_{\mathrm{BH}}$.

${ }^{c}$ Black hole radiative efficiency.

${ }^{d} \mathrm{BAL}$ wind momentum loading defined such that $\eta_{p}=\dot{p}_{\mathrm{BAL}} /(L / c)$.

${ }^{e} \mathrm{BAL}$ wind energy loading defined such that $\eta_{E}=\dot{E}_{\mathrm{BAL}} / L$.

overwhelming majority of the energy budget remains in the particle's kinetic energy.

After the BAL wind particles are spawned, they are treated identically to all other gas in the simulation. Depending on the local ISM gas geometry and BAL wind particle trajectory, the BAL winds may either interact hydrodynamically with the ambient ISM gas or vent through low-density channels.

\subsubsection{BAL wind speeds}

In all simulations presented in Section 3, we hold fixed the value for the BAL wind mass loading at $\eta_{\mathrm{BAL}}=1$ (or equivalently $f_{\text {acc }}=0.5$ ) and vary only the BAL input wind speed. In addition to a no BAL feedback simulation, we adopt three wind speed values of $v_{\mathrm{BAL}}=$ $\{3000,10000,30000\} \mathrm{km} \mathrm{s}^{-1}$. Varying the wind speed changes both the direct momentum and direct energy injection from the AGN (see equations 4 and 5). The $v_{\mathrm{BAL}}=3000 \mathrm{~km} \mathrm{~s}^{-1}$ wind is therefore weaker in both energy and momentum injection compared with the faster wind simulations. We therefore refer to the $v_{\mathrm{BAL}}=3000 \mathrm{~km} \mathrm{~s}^{-1}$ wind speed simulation as the 'weak' wind simulation; the $v_{\mathrm{BAL}}=$ $10000 \mathrm{~km} \mathrm{~s}^{-1}$ wind speed simulation as the 'intermediate' wind simulation; and the $v_{\mathrm{BAL}}=30000 \mathrm{~km} \mathrm{~s}^{-1}$ wind speed simulation as the 'strong' wind simulation in Section 3. The weak, intermediate, and strong wind simulations contain energy loading factors of $\eta_{E}=$ $\{0.0005,0.005,0.05\}$, respectively. A summary of the different wind speed runs, along with a summary of additional BAL wind feedback parameters, can be found in Table 1 .

\subsubsection{Compton heating and cooling}

Compton heating and cooling from the AGN is followed explicitly in the simulations. As argued in Sazonov, Ostriker \& Sunyaev (2004), we assume the AGN radiation field has a nearly obscurationindependent Compton temperature $T_{\text {Compt }} \approx 2 \times 10^{7} \mathrm{~K}$. The gas Compton heating/cooling rate for gas with temperatures $T<10^{9} \mathrm{~K}$ is then calculated as

$\Lambda_{\mathrm{Compt}}=\frac{4 k_{\mathrm{B}}\left(T_{\mathrm{Compt}}-T\right)}{m_{\mathrm{e}} c^{2}} \frac{L_{\mathrm{AGN}} \sigma_{\mathrm{T}}}{4 \pi r^{2}} n_{\mathrm{e}}$,

where $T$ is the gas temperature, $m_{\mathrm{e}}$ the electron mass, $\sigma_{\mathrm{T}}$ the Thompson cross-section, $n_{\mathrm{e}}$ the electron number density (bound + free), $L_{\mathrm{AGN}}$ the bolometric AGN luminosity, and $r$ the distance of a gas element from the AGN. Hotter gas (i.e. $T>10^{9} \mathrm{~K}$ ) is treated with the appropriate relativistic form of the Compton heating/cooling rates. Note that here we have used the optically thin expression $L_{\mathrm{AGN}} /\left(4 \pi r^{2} c\right)$ for the radiation energy density incident from the AGN; this also follows from Sazonov et al. (2004) who show that even deeply obscured AGN (with all UV/optical downgraded to 
Table 2. Initial conditions of the galaxies simulated in this paper.

\begin{tabular}{lcccccccccccc}
\hline IC & $\begin{array}{c}L_{\mathrm{Edd}}{ }^{a} \\
\left(\mathrm{erg} \mathrm{s}^{-1}\right)\end{array}$ & $\begin{array}{c}M_{\text {total }}{ }^{b} \\
\left(\mathrm{M}_{\odot}\right)\end{array}$ & $\begin{array}{c}M_{\mathrm{disc}}{ }^{c} \\
\left(\mathrm{M}_{\odot}\right)\end{array}$ & $\begin{array}{c}M_{\text {bulge }}{ }^{d} \\
\left(\mathrm{M}_{\odot}\right)\end{array}$ & $\begin{array}{c}M_{\mathrm{BH}}{ }^{e} \\
\left(\mathrm{M}_{\odot}\right)\end{array}$ & $M_{\mathrm{BH}} / M_{\text {bulge }}{ }^{f}$ & $f_{\mathrm{g}, \text { init }}{ }^{g}$ & $\mathrm{CC}^{h}$ & $\Lambda^{i}$ & $\begin{array}{c}h^{j} \\
(\mathrm{kpc})\end{array}$ & $\begin{array}{c}\epsilon_{\text {soft }}{ }^{k} \\
(\mathrm{pc})\end{array}$ & $\begin{array}{c}m_{\mathrm{b}}{ }^{l} \\
(\mathrm{M} \odot)\end{array}$ \\
\hline $\mathrm{G} 3$ & $1.0 \times 10^{45}$ & $8.2 \times 10^{11}$ & $2.9 \times 10^{10}$ & $6.5 \times 10^{9}$ & $8.2 \times 10^{6}$ & $1.3 \times 10^{-3}$ & $20 \%$ & 6.0 & 0.05 & 1.8 & 1.0 & $2 \times 10^{3}$ \\
$\mathrm{G} 4$ & $1.3 \times 10^{46}$ & $2.4 \times 10^{12}$ & $6.2 \times 10^{10}$ & $6.2 \times 10^{10}$ & $1 \times 10^{8}$ & $1.6 \times 10^{-3}$ & $20 \%$ & 5.0 & 0.05 & 3.8 & 1.0 & $5 \times 10^{3}$ \\
$\mathrm{G} 4$ ×3 & $3.8 \times 10^{46}$ & $2.4 \times 10^{12}$ & $6.2 \times 10^{10}$ & $6.2 \times 10^{10}$ & $3 \times 10^{8}$ & $4.8 \times 10^{-3}$ & $20 \%$ & 5.0 & 0.05 & 3.8 & 1.0 & $5 \times 10^{3}$ \\
G4_x10 & $1.3 \times 10^{47}$ & $2.4 \times 10^{12}$ & $6.2 \times 10^{10}$ & $6.2 \times 10^{10}$ & $1 \times 10^{9}$ & $1.6 \times 10^{-2}$ & $20 \%$ & 5.0 & 0.05 & 3.8 & 1.0 & $5 \times 10^{3}$ \\
\hline
\end{tabular}

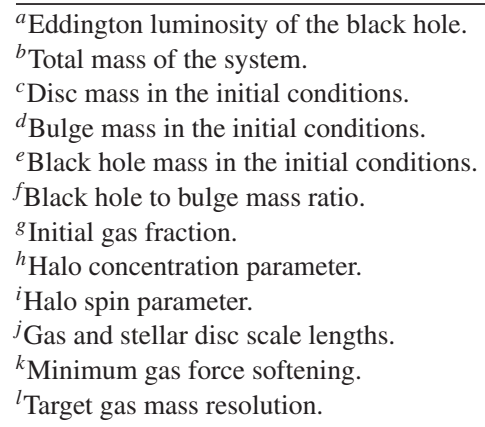

infrared) produce nearly identical Compton cooling rates, while the Compton heating is dominated by very hard X-ray photons for which the resolved obscuration in our simulations is negligible.

In addition, as has been discussed in Faucher-Giguère \& Quataert (2012), while electrons in the post-shock gas may be able to efficiently cool via Compton cooling off the AGN radiation, the low densities of the post-shock BAL gas limits the efficiency with which protons and electrons remain in temperature equilibrium. This can lead to the creation of a two-temperature plasma, resulting in a population of cool electrons and significantly hotter protons. In our model, the proton and electron temperatures are not tracked independently. Instead, since the protons are the dynamically important species, the gas temperature that we track in the code is the proton temperature. We therefore implement a cooling rate limiter to prevent the protons from cooling more quickly than they can transfer energy to the ambient electrons. In practice, we limit the gas cooling rate using the Coulomb collision cooling rate limiter described in Richings \& Faucher-Giguère (2018b) where we assume that the electrons have a temperature of $T=2 \times 10^{7} \mathrm{~K}$ and the protons are at the current gas temperature. However, we have verified that the (very hot) postshock gas in our simulations (specifically, the directly injected wind particles) radiatively cools negligibly before leaving the galaxy, and therefore the exact implementation of this cooling procedure is not critical to the results presented in this paper.

\subsection{Isolated galaxy initial conditions}

We construct initial conditions that are nearly identical to those employed in Torrey et al. (2017), which is based on the method outlined in Springel, Di Matteo \& Hernquist (2005), with a few minor changes and extensions as described below. Each initial galaxy consists of a gas disc, stellar disc, stellar bulges, central supermassive black hole, and dark matter halo. The gas and stellar discs are taken to have exponential profiles $\left(\Sigma=\Sigma_{0} \exp (-r / h)\right)$ with initially identical disc scale lengths (i.e. $h_{\text {gas }}=h_{\text {stars }}$ ). The stellar bulge and dark matter halo are both modelled as a Hernquist (1990) profile ( $\rho=M a / 2 \pi r(r$ $+a)^{3}$ ), but with independent scalings. The central supermassive black hole is placed initially at the centre of each galaxy. There is no hot halo included in our initial set-up.

We employ two initial galaxies (G3 and G4) that are constructed to roughly follow empirically derived scaling relations for low-redshift galaxies, meaning that higher mass galaxies tend to have lower gas fractions, higher bulge fractions, smaller halo concentration values, and larger disc scale lengths. A summary of the initial condition galaxy parameters is given in Table 2 . In addition, several variations of the G4 galaxies are run with larger black hole masses. Since all simulations use prescribed Eddington accretion rate black holes, the modified black holes masses sample the impact of increasing the black hole feedback strength without changing the surrounding galaxy's properties.

The gas distribution in the initial conditions is smooth (not multiphase) and non-turbulent. This forces the initial conditions to go through a relaxation phase after the simulation is started, where dense gas clumps form, stars form, and ultimately turbulence begins to be driven via stellar feedback. To accommodate this relaxation period, we evolve all of our isolated disc galaxies in time for 1 Gyr, at which point we find they have achieved a (roughly) steady state in their SFRs and ISM phase structure. All simulations presented in this paper employ these 'relaxed' initial conditions. This relaxation period changes the gas fractions downward by $\sim 5$ per cent in both simulations since gas is consumed by star formation but not replenished. Images of the gas distribution for the relaxed G4 initial conditions used in this paper are shown in Fig. 1. Owing to their long relaxation periods, a multiphase and self-regulating ISM is present which is important for when the AGN winds begin to operate.

\section{IMPACT OF BAL WINDS ON HOST GALA XIES}

In this section, we consider the impact of the BAL winds on their host isolated disc galaxies. We specifically consider the impact of different BAL wind strengths (as described in the previous section and summarized in Table 1).

\subsection{Central gas cavity geometry}

Fig. 2 shows time series images of the gas distribution in the G3 disc evolved without (top) and with (bottom three rows) BAL feedback. Each time series shows the evolution of the gas distribution within the central $2 \mathrm{kpc}$ evolved between 10 and $50 \mathrm{Myr}$ to demonstrate the gas reaction to the BAL feedback. All panels are arranged to place the black hole at the centre of the field of view. From top to 

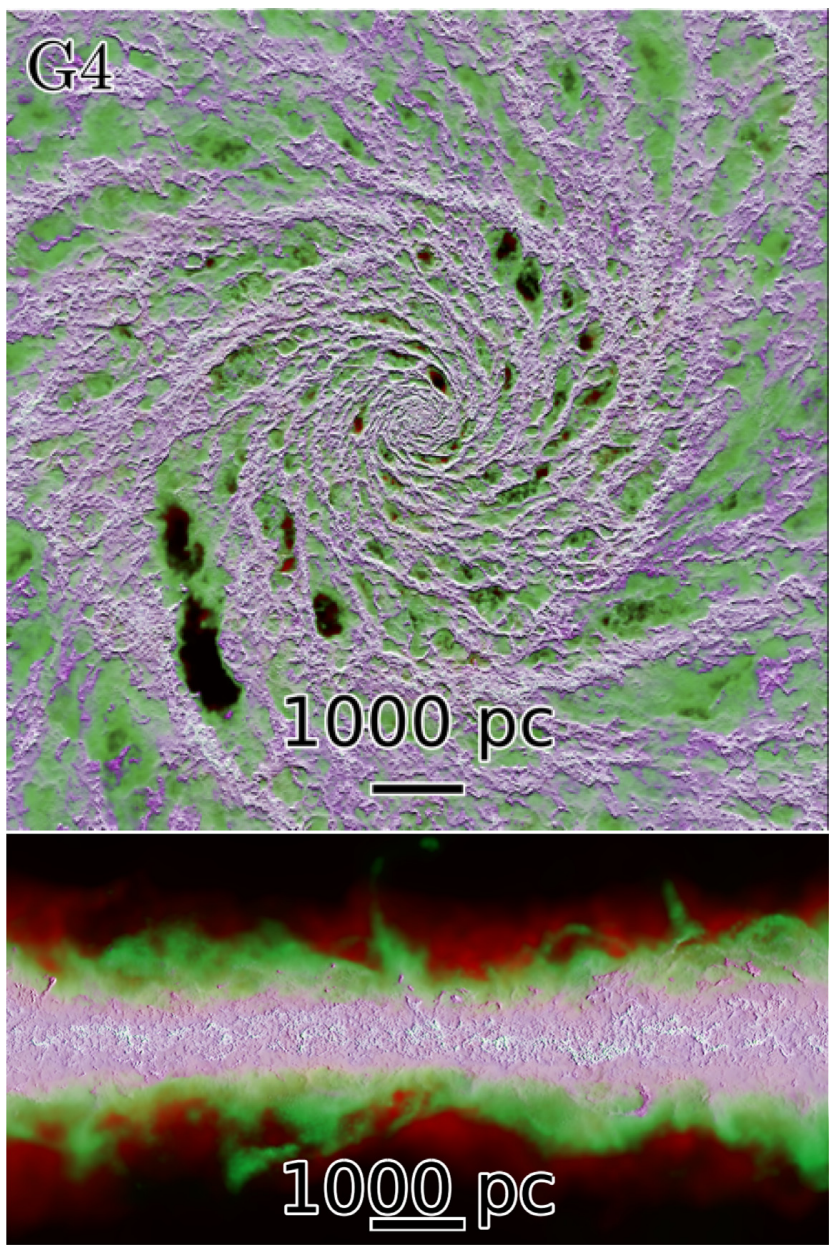

Figure 1. Gas distribution for the G4 initial condition galaxy used in this paper. Colour indicates gas temperature with magenta being cool/cold gas (i.e. $T<10^{4} \mathrm{~K}$ ) and green being warm/ionized gas (i.e. $10^{4}<T<10^{6} \mathrm{~K}$ ), while pixel brightness indicates total gas column density. The gas distributions shown here are the result of evolving each galaxy in isolation, without any black hole feedback, for 1 Gyr to allow a multiphase ISM structure to develop under the influence of stellar feedback.

bottom, the simulations show the no feedback, weak, intermediate, and strong BAL winds (see Section 2.2.3 for definitions).

In the no BAL feedback case, the evolution of the central gas reservoir is driven by gas dynamics in conjunction with stellar feedback. A multiphase ISM is sustained with dense and cold starforming gas pockets (indicated with magenta within Fig. 2) forming within a warm, volume-filling gas (indicated with green and red in Fig. 2). As explored in detail in Torrey et al. (2017), the central gas mass undergoes an episodic evolution pattern - including periods of low and high central gas densities - that is driven by an unstable equilibrium between turbulent gas energy dissipation and turbulent energy injection via stellar feedback in short dynamical time regions. Feedback from star formation alone can be responsible for lowering the central gas density, but gas is able to return to the central region in $\sim$ tens of Myr. Though not shown in this figure, the episodic central gas mass cycle manifests in the top row time series of Fig. 2 via a low central gas density at $t=20 \mathrm{Myr}$ (Torrey et al. 2017).

The behaviour of the simulations with BAL winds included (bottom three panels of Fig. 2) have more sustained and pronounced central gas cavities when compared with the no BAL wind simulation. The size of the central gas cavity increases with the strength

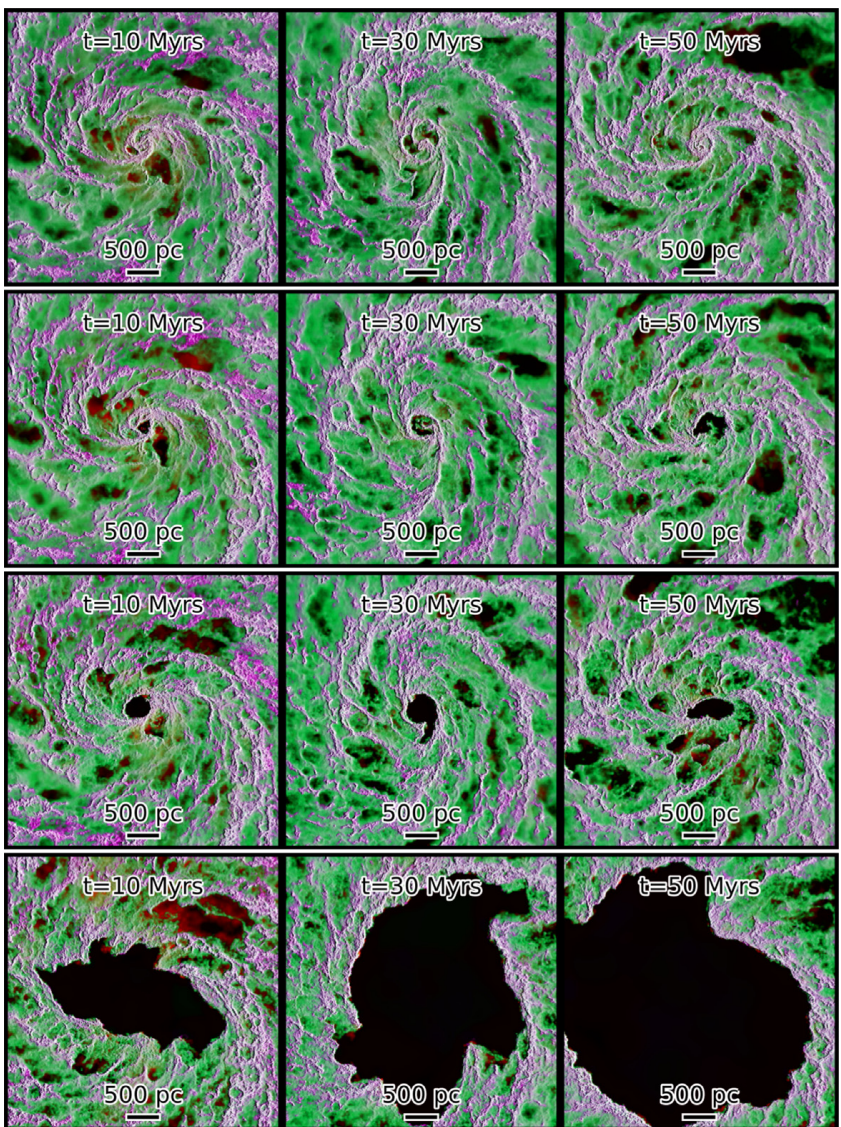

Figure 2. Time series of face-on gas projections of the G3 disc with no BAL feedback (top), weak winds (second row), intermediate winds (third row), and strong winds (bottom). Times and scale bars are marked within. The size of the central cavity increases with the velocity/strength of the BAL wind material, reaching $\sim 1 \mathrm{kpc}$ in the strongest wind simulation. Magenta indicates cold gas $(\sim 100 \mathrm{~K})$, green indicates warm/ionized material $\left(\sim 10^{4} \mathrm{~K}\right)$, and red indicates hot gas $\left(\sim 10^{6} \mathrm{~K}\right)$. The brightness or opacity of each pixel indicates the total column density. Despite being hot, the central gas cavities appear mostly dark because the gas densities are very low.

of the AGN feedback. The gas distribution at large (i.e. $\sim 1 \mathrm{kpc}$ ) distances from the black hole is not significantly changed in the weak wind simulation (compare, e.g. the off-centre gas distribution of the top three rows at $t=30 \mathrm{Myr}$ ). Instead, the majority of the impact from the weak BAL winds s confined to the central $\sim 100 \mathrm{pc}$, where the central gas density is reduced. Examining the intermediate and strong BAL wind speed cases reveals qualitatively similar behaviour, but with the central gas cavity now being increasingly well defined, and marginally (significantly) larger for the intermediate (strong) BAL wind strength.

The central gas cavity is a natural consequence of the BAL wind feedback that we have implemented in this paper. For a fast outflow expanding into a static ambient medium the expected outflow solution has been explored in detail (King \& Pounds 2003; FaucherGiguère \& Quataert 2012). The post-shock wind material is expected to be highly overpressurized when compared against the ambient ISM (at least when the hot gas is effectively confined). The central low-density gas cavity is created as the overpressurized post-shock gas expands and pushes upon the ambient ISM.

The pressure of the post-shock gas is largely set by the BAL wind properties that we have manually chosen for our simulations. In the idealized spherically symmetric case, the average density of the 
post-shock wind material, $n_{\mathrm{sw}}$, depends on the initial properties of the BAL winds along with radius according to

$n_{\mathrm{sw}} \approx \frac{\eta_{\mathrm{BAL}} \dot{M}_{\mathrm{BH}}}{\pi r^{2} v_{\mathrm{BAL}} m_{\mathrm{p}}}$

$\approx \frac{0.2}{\mathrm{~cm}^{3}} \eta_{\mathrm{BAL}}\left(\frac{M_{\mathrm{BH}}}{10^{8} \mathrm{M}_{\odot}}\right)\left(\frac{v_{\mathrm{BAL}}}{0.1 c}\right)^{-1}\left(\frac{r}{100 \mathrm{pc}}\right)^{-2}$.

Assuming a strong shock of velocity, $v_{\mathrm{s}}$, in a monatomic gas, the Rankine-Hugoniot jump conditions give the post-shock gas temperature as

$T=\frac{3 \mu}{16 k} m_{\mathrm{p}} v_{\mathrm{s}}^{2} \approx 1.2 \times 10^{10}\left(\frac{v_{\mathrm{s}}}{0.1 c}\right)^{2} \mathrm{~K}$.

Assuming that $v_{\mathrm{s}} \approx v_{\mathrm{BAL}}$, this leads to an order-of-magnitude expected post-shock gas pressure of

$P \sim \frac{2.4 \times 10^{10} \mathrm{~K}}{\mathrm{~cm}^{3}} \eta_{\mathrm{BAL}}\left(\frac{M_{\mathrm{BH}}}{10^{8} \mathrm{M}_{\odot}}\right)\left(\frac{v_{\mathrm{BAL}}}{0.1 c}\right)\left(\frac{r}{100 \mathrm{pc}}\right)^{-2}$,

which can be very large when compared against typical solar neighbourhood ISM pressures of $P_{\text {ISM }} \sim 10^{4}-10^{5} \mathrm{~K} \mathrm{~cm}^{-3}$.

Detailed studies of the interaction of fast winds with an ambient static material reveal a clear prediction for a dual-shock structure accompanied with a contact discontinuity separating the two fronts (e.g. King \& Pounds 2003; Faucher-Giguère \& Quataert 2012). While we can easily identify the bulk properties of both ISM and wind-shocked material, isolating and demonstrating that the detailed shock structure is recovered in our simulations is not easily done owing to (i) the very low density (and therefore poorly sampled) nature of the wind material, which results in noisy shock structure diagnostics, and (ii) significant deviations from clean/symmetrical geometry such that any features tend to get blurred out. We stress, however, that what is likely most critical for the dynamical impact of feedback is capturing shock heating of the small-scale wind, which produces the hot, energy-conserving wind bubble. The fact that the wind bubbles in our simulations are filled with hot gas with temperature in good agreement with the shock jump condition in equation (11), as well as the insensitivity of the results to the mass of the spawned with resolution elements, suggest that this shock heating is adequately captured by the hydro solver.

The central gas cavities identified in Fig. 2 are opened by the pressure difference that exists between the heated BAL wind material and the surrounding ISM. In weak and intermediate BAL wind strength simulations, the BAL-driven central gas cavity initially grows in size, but stalls after 20-30 Myr. The strong BAL simulation still shows signs of cavity growth (albeit slow) at 40 Myr. Qualitatively similar results are found for the other initial condition galaxies that are used in this paper but not shown in Fig. 2. The exact size of the cavity and time-scale over which the cavity evolves are functions of the host galaxy properties, BAL wind speed, and the AGN luminosity.

Fig. 3 shows cylindrical projections of the mass-weighted average gas density (left), mass-weighted average gas temperature (middleleft), mass weighed averaged gas thermal pressure (middle-right), and mass-weighted average gas speed (right) for the G3 galaxy at $t=20$ Myr while AGN feedback is actively carving out the central low-density cavity. The top row shows the result from the simulation without BAL winds, while the remaining bottom three rows show the simulations with weak, intermediate, and strong BAL winds in the second, third, and fourth rows, respectively. The cylindrical projections reveal more directly the mechanics of the BAL wind coupling. The cylindrically averaged gas density (left-hand column) shows that while the cavity size is not dramatically changed at these early stages of cavity expansion, the faster wind speed simulations have a significantly stronger impact on the gas above and below the disc plane. The gas density in all of the simulations (including the no BAL feedback simulations) has a polar angular dependence with the densest gas being restricted to the disc plane by construction based on our employed isolated disc initial conditions. The BAL winds drive outflows preferentially in the polar directions that lowers the gas density. The confinement of the BAL wind outflows is naturally shaped by the surrounding galactic gas and dependent on the BAL wind speed with the high-velocity winds having a larger outflow channel that drives down the gas density for a larger region off the galaxy disc plane.

The temperature map (middle-left column) for the no BAL simulation shows that most of the ISM gas is at a mass-weighted temperature of $T \lesssim 10^{4} \mathrm{~K}$, with material off the disc plane being somewhat hotter at $\sim 10^{6} \mathrm{~K}$. In stark contrast, the gas that has been impacted by the BAL winds is very clearly identified in the bottom three temperature maps. A hot gas channel is found around the central black hole that extends in the polar direction. The size of the hot gas channel expands further away from the disc plane. The temperature of the hot gas depends directly on the input BAL wind speed, as described in equation (11), and as will be explored in subsequent plots.

The pressure maps (middle-right column) for all three simulations indicate there is a pressure jump in the galaxy disc plane between the central low-density, high-temperature cavity gas and the ambient ISM. The disc-plane pressure jump is reasonably sharp in these projections that is notable given that the cylindrical projections tend to blur any features that are not exactly cylindrically symmetric. The disc-plane pressure jump corresponds to the forward shock, where the fast wind material and shock-heated ISM make contact with the unshocked/ambient ISM. Off the disc plane, there is a steep but smooth pressure gradient. The reverse-shocked, hot BAL wind material is able to either do work on the ISM or move out of the disc plane by accelerating in the polar directions.

Whereas the gas temperature associated with BAL wind material is visibly different between the different BAL wind strength runs, the central gas pressure is reasonably similar. The similarity of the central gas pressures is consistent with the weaker scaling of the pressure with wind speed as given in equation (12), as compared to the post-shock gas temperature. Specifically, while the post-shock gas temperature jumps significantly with higher wind speed $(T \propto$ $\left.v_{\mathrm{s}}^{2}\right)$, the central gas density decreases at the same time $\left(\rho \propto v_{\mathrm{s}}^{-1}\right)$. As a result, the central gas pressure in the strong wind simulation has only a marginally higher central gas pressure compared with the weaker wind simulations.

The gas speed map (right-hand column) for the no BAL wind simulation is mostly smooth, with minor indications of faster material being found in the polar directions. The lack of strong features in the no BAL simulation velocity map is a result of the modest outflow velocities associated with stellar feedback alone. In contrast, the three BAL wind simulations indicate that the low-density material in the central cavity and in the polar directions retains velocities significantly higher than the ambient ISM. The higher gas speeds in the polar directions - which are significant fractions of the initial input wind velocity - are consistent with much of the BAL wind material venting through these polar channels. The speed found in the outflows scales directly with the BAL manually imposed wind input velocity.

Since the BAL wind particles are spawned isotropically and initially propagate in the radially outward direction from the central black hole, interaction with the dense ISM is not guaranteed. The opening angle for the ISM disc - including the somewhat 


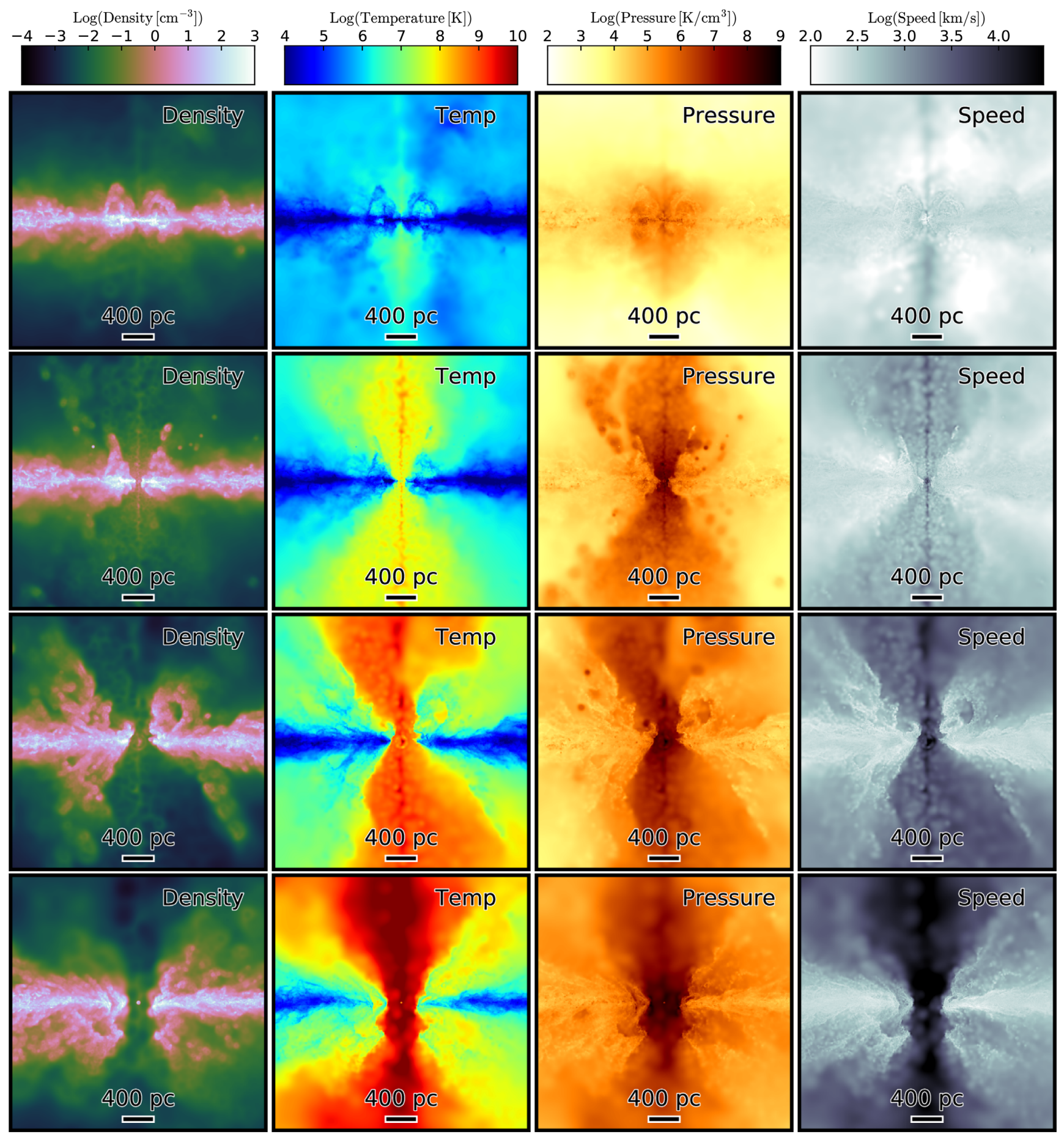

Figure 3. Cylindrical projections of the gas density (left-hand column), mass-weighted average gas temperature (centre left), mass-weighted average gas thermal pressure (centre right), and mass-weighted average gas speed (right-hand column) are shown for the no feedback (top row), weak (second row), intermediate (third row), and strong (bottom row) feedback simulations at $t=20$ Myr for the G3 disc. The cavity size and clearing of off-disc-plane gas both increase with faster BAL winds. However, in all cases the same qualitative picture for the BAL wind coupling mechanics is present. In the disc plane, there is a sharp pressure jump between the post-shock BAL wind material and the ambient ISM. Out of the disc plane there is a steep but smooth pressure gradient that allows material to be accelerated, while the highest velocity outflows vent in the polar directions.

warm/diffuse gas around the disc mid-plane - as seen from the black hole's position changes with the adopted BAL wind speed, and can become quite small for the strong feedback simulation. The reduced opening angle of the ISM disc as seen by the central black hole results in a significant fraction of wind material directly venting from the black hole through the polar low-density channels without strongly interacting with the ISM disc. The interaction between the BAL winds and the ISM must therefore take into account a geometric feedback coupling efficiency that describes the fraction of BAL wind material that will interact with the ISM. The geometric feedback 

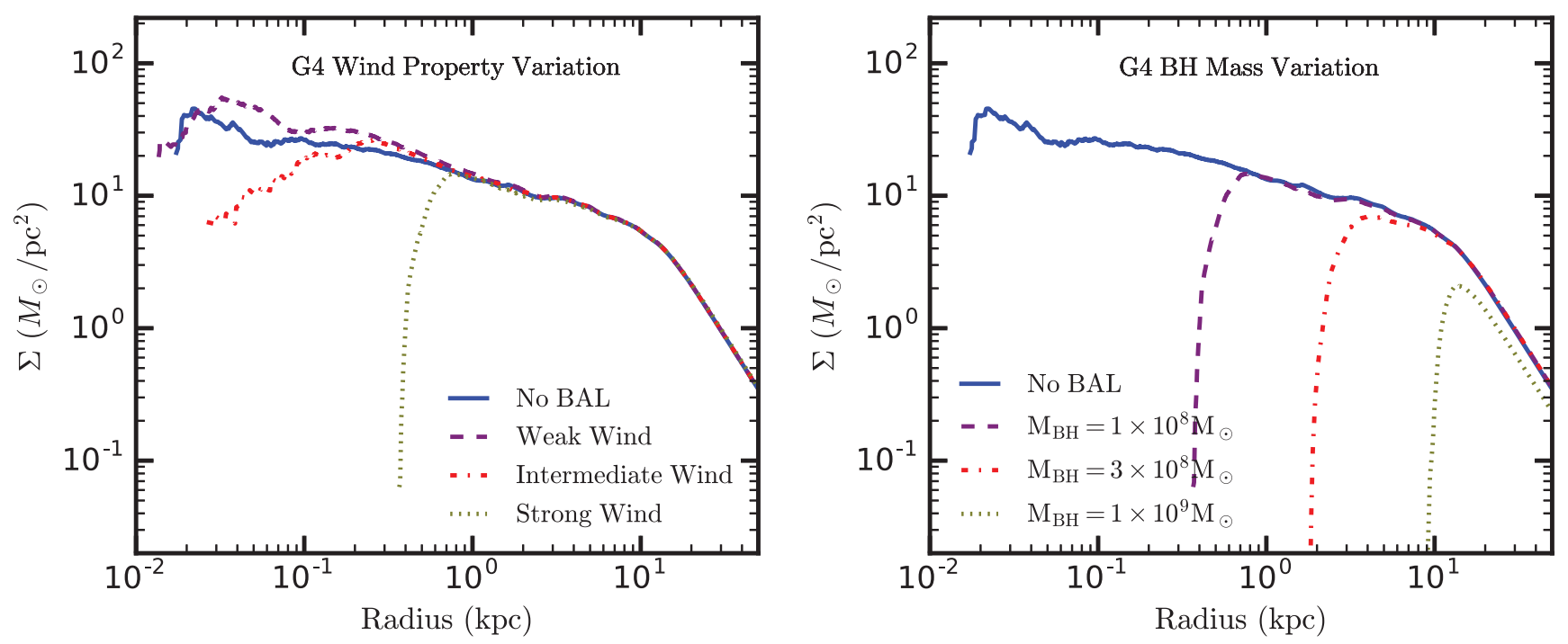

Figure 4. Gas surface density profiles for G4 are shown with the lines indicating the results for the varied BAL feedback strengths at a fixed black hole mass (left) and varied black hole masses with strong winds (right) at $t=20 \mathrm{Myr}$. BAL wind material is neglected when constructing the gas density profiles. The central gas density drops in the simulations including BAL feedback and the size of the central gas cavity increases with either increased BAL wind strength (left) or with increased BH luminosity (right). We adopt a cavity surface density as $\Sigma_{\text {gas }}=10 \mathrm{M}_{\odot} \mathrm{pc}^{-2}$ to define the size of the central cavity in subsequent analysis. Owing to the sharp drop in the gas surface density in the BAL feedback simulations, the calculated cavity size is not very sensitive on the specified threshold density chosen.

coupling efficiency is generically small for isolated galaxies with reasonably thin gas discs, and decreases further as the central lowdensity cavity grows in size.

\subsection{Impact on host galaxy gas}

The left-hand panel of Fig. 4 shows surface density profiles for the G4 galaxy at $t=20 \mathrm{Myr}$. Each line indicates the result from a different BAL wind speed, as indicated in the legend, with the blue line being the no feedback profile. At large distances (i.e. $r \gtrsim$ $2-3 \mathrm{kpc}$ ) the gas surface density profiles agree almost identically regardless of the BAL feedback strength. This agreement indicates that the BAL feedback has a limited impact on the galactic disc outside of the central $\sim \mathrm{kpc}$. The exact radius beyond which the BAL feedback is inefficient at modifying the gas surface density profile changes with galaxy properties and depends on the BAL wind strength and luminosity. As the BAL wind strength is increased, the central cavity size tends to also increase, as was indicated to be the case in Fig. 3. The right-hand panel of Fig. 4 shows the same quantities, but for the G4 galaxy with strong winds and varied black hole masses. Perhaps unsurprisingly, we find that increasing the black hole mass - and therefore the black hole luminosity allows for further growth of the central cavity. For largest black holes considered here $\left(M_{\mathrm{BH}}=10^{9} \mathrm{M}_{\odot} ; L_{\mathrm{AGN}} \approx 10^{47} \mathrm{erg} \mathrm{s}^{-1}\right)$, the cavity size is nearly $\sim 10 \mathrm{kpc}$, which covers most of the star-forming gas in the galaxy. For these most luminous systems that we simulate, we find that the feedback is indeed capable of having a very significant impact on the host galaxy in spite of the inefficient geometri c coupling.

We note that the fiducial black hole mass used in our G4 disc results in a black hole to bulge mass ratio of $M_{\mathrm{BH}} / M_{\text {bulge }}=1.6 \times 10^{-3}$ (a central value in the black hole to bulge mass ratio distribution; $L_{\mathrm{AGN}} \approx 10^{46} \mathrm{erg} \mathrm{s}^{-1}$ ), while the most massive black hole in the G4 disc has a value of $M_{\mathrm{BH}} / M_{\text {bulge }}=1.6 \times 10^{-2}$ (which is reasonable, but closer to the upper end of the distribution; $L_{\mathrm{AGN}} \approx 10^{47} \mathrm{erg} \mathrm{s}^{-1}$ ). It is worth noting that our G3 and G4 systems with fiducial black hole to bulge mass ratios of $\sim 10^{-3}$ have a limited impact on their host galaxy. However, at the same time we find that increasing the black hole mass within the scatter in the $M_{\mathrm{BH}} / M_{\text {bulge }}$ value distribution can lead to a significantly increased impact on the host galaxy.

Using Fig. 4 as motivation, we define a 'central gas cavity size' as the radius at which the average surface density drops below $\Sigma_{\text {gas }}=$ $10 \mathrm{M}_{\odot} \mathrm{pc}^{-2}$ not including BAL wind material. When a disc has a density profile that does not exceed this surface density value, we adopt the radius of maximum surface density as the cavity radius. The resulting cavity size measurements are fairly robust for most of the strong feedback cases, but can in some cases yield ill-defined cavity sizes for the no feedback and weak feedback runs. Owing to the steepness of the surface density profiles in most of the BAL wind runs, our cavity size definition is not strongly impacted by changes in the specific gas surface density threshold by a factor of a few in either direction.

Fig. 5 shows the cavity size as a function of time using the $\Sigma_{\text {gas }}=$ $10 \mathrm{M}_{\odot} \mathrm{pc}^{-2}$ surface density threshold. For the no BAL feedback case (blue line), we do not find evidence for a sustained/resolved central cavity. In contrast, all of the intermediate and strong BAL wind simulations show a very clear sustained central gas cavity. The size of the central gas cavity is larger for the strong BAL wind simulations reaching a size of $\sim 1.5 \mathrm{kpc}$. For the weak and intermediate feedback strengths the cavity size rapidly expands initially, and then stalls after only 10-20 Myr. For the strong BAL wind simulations the cavity expands rapidly upon start up, but in general continues to experience slowed expansion through the first $50 \mathrm{Myr}$.

We note here, but discuss further in Section 5.2, that the results presented in this section consider only constant accretion rates. It is reasonable to speculate that the black hole accretion rates will be significantly self-regulated once a sizable central gas cavity is created. Thus, the cavity sizes explored in this section likely overestimate that which would result in models that include selfconsistently determined accretion rates. This is especially when the cavity sizes become comparable in size to the galaxy as a whole. 


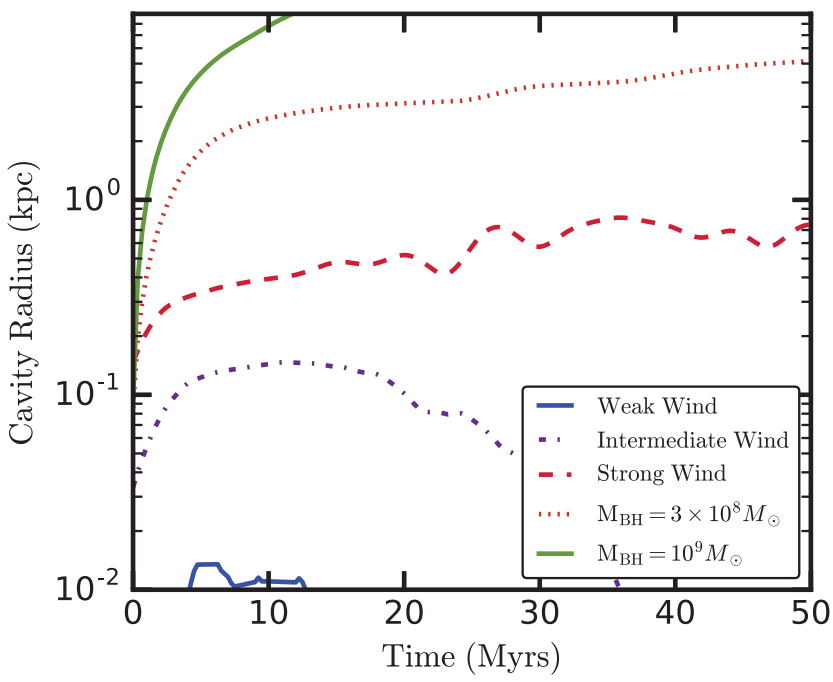

Figure 5. Central gas cavity size versus time for the G4 galaxy. Cavity size is evaluated as the distance within which the gas surface density profile falls below $\Sigma_{\text {gas }}=10 \mathrm{M}_{\odot} \mathrm{pc}^{-2}$ (see Fig. 4 and associated caption). For the weak BAL feedback simulations the central gas cavity size is small in value and noisy in its evolution. The noisy central cavity size is driven in part by the bursty stellar feedback behaviour and movement of the black hole. For the intermediate and strong BAL feedback cases, a clear and well defined cavity emerges that evolves smoothly with time. In all cases, however, the cavity slows/stalls its expansion after only $\sim 10 \mathrm{Myr}$.

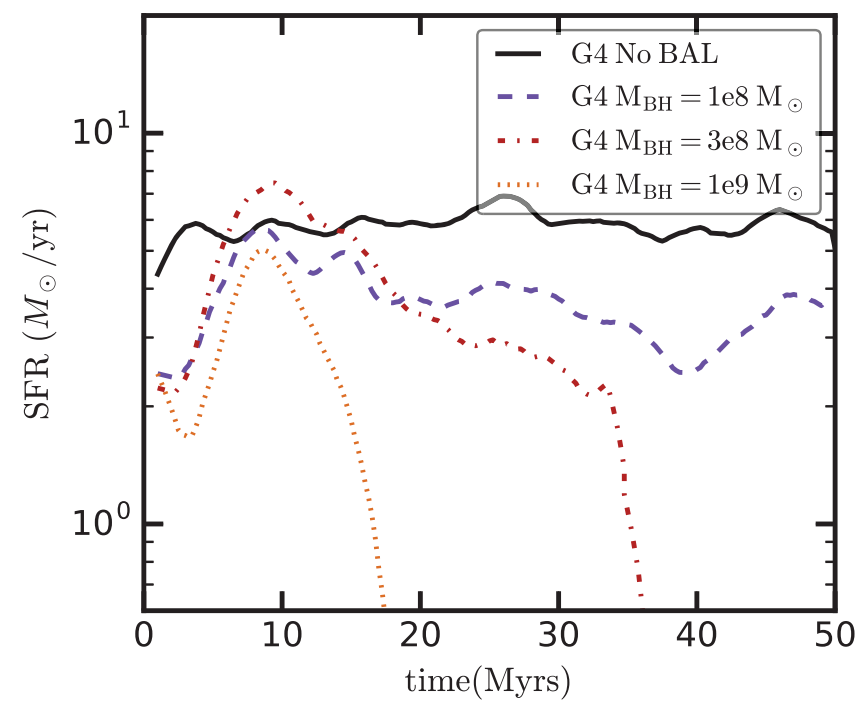

Figure 6. Star formation rates (SFRs) for the G4 disc with strong winds and varied black hole masses (i.e. resulting in varied AGN luminosity). The SFRs for the lowest mass black hole is not strongly impacted by the BAL feedback (i.e. it is reduced only by a factor of only $\sim 20$ per cent) owing to the relatively centrally concentrated nature of the BAL wind coupling. SFRs are significantly impacted for the most luminous AGN, and can be suppressed in $\sim 20-30$ Myr for the most massive black holes in the strong wind case.

\subsection{Impact on host galaxy SFR}

Having explored the impact of the AGN fast winds on the central gas distribution, we finally consider the global SFRs for varied black hole masses (and therefore varied AGN luminosities) in Fig. 6. The impact of the BAL winds on the global SFR is limited for the lowest black hole mass. This is a result of the localized impact of the BAL winds on the host galaxy. From Fig. 5, the weak, intermediate, and even the strong BAL wind runs all show maximum cavity sizes of $\sim 1 \mathrm{kpc}$. Examining Fig. 1, the majority of star-forming gas falls outside of the central $\sim 1 \mathrm{kpc}$, which is not strongly impacted by the BAL feedback.

We note that this result is somewhat surprising because only the central few kpc of ISM gas are being depleted despite the presence of a powerful AGN. Even though the lowest black hole mass simulation has a black hole to bulge mass ratio of $M_{\mathrm{BH}} / M_{\text {bulge }} \sim 10^{-3}$, it still includes an Eddington accretion rate black hole injecting feedback with the fiducial/nominal AGN efficiency. Yet, it is only strongly impacting the central $\sim 1 \mathrm{kpc}$ of ISM gas over the first $50 \mathrm{Myr}$, and accordingly only modestly impacting the SFR. Our simulations specifically indicate that isolated galaxies with relatively thin gas discs and modest black hole to bulge mass ratios may be able to host luminous AGN activity without undergoing significant star formation suppression. The inefficiency of AGN feedback in our simulations stems directly from the inefficient geometric feedback coupling that occurs in disc galaxies as the central cavity grows.

There are, however, ways to increase the impact of AGN feedback including further increases to the black hole mass or equivalently AGN luminosity. Fig. 6 shows the SFRs as a function of time for the G4 disc with strong winds and increased black hole masses (see Table 2). For our fixed Eddington accretion rate simulations, increasing the black hole mass has the direct consequence of increasing the AGN luminosity that leads to further suppression of the SFR. We find that the simulation with most massive black hole (G4 with a black hole mass of $M_{\mathrm{BH}}=10^{9} \mathrm{M}_{\odot}$ ) shuts down the SFR in $\sim 20$ Myr. For this simulation, the bulge to black hole mass ratio is $\sim 50$. Progressing down in black hole mass, the less luminous AGN still suppress the host galaxy SFR somewhat, but only at the factor of a few level. Our simulations therefore indicate fast AGN winds can have a significant impact on the global SFR in isolated disc galaxies, but the low geometric feedback coupling efficiency limits the strong impact to comparatively massive black holes with sustained high accretion rates.

Though we do not explore it in this paper, there are other possible ways to increase the AGN feedback impact, including by changing the gas geometry around the central black hole. The low AGN feedback efficiency seen in the majority of runs presented in this paper is a product of the low coupling efficiency to the employed isolated disc galaxies that allow for efficient wind venting in the polar directions. Galaxies with very thick gas discs (e.g. as might be common at high redshift) or with disturbed/perturbed central gas distributions (e.g. as is associated with galaxy merger events) would not necessarily suffer from low feedback coupling efficiencies. Further, although we do not explore it in this paper, we discuss the possibility that disturbed gas distributions around the central AGN may aid in driving large/rapid outflows.

\section{PROPERTIES OF WINDS}

In this section, we explore the properties of the outflow material for the same simulations presented in the previous section. We consider the gas radial velocity distribution, distribution of gas in densitytemperature phase space, and total momentum in the outflows.

\subsection{Radial velocity distribution}

Fig. 7 shows the gas radial velocity distribution for the G4 disc with the lines corresponding to the three different wind speed simulations along with the no BAL wind simulation, as indicated in the legend. 


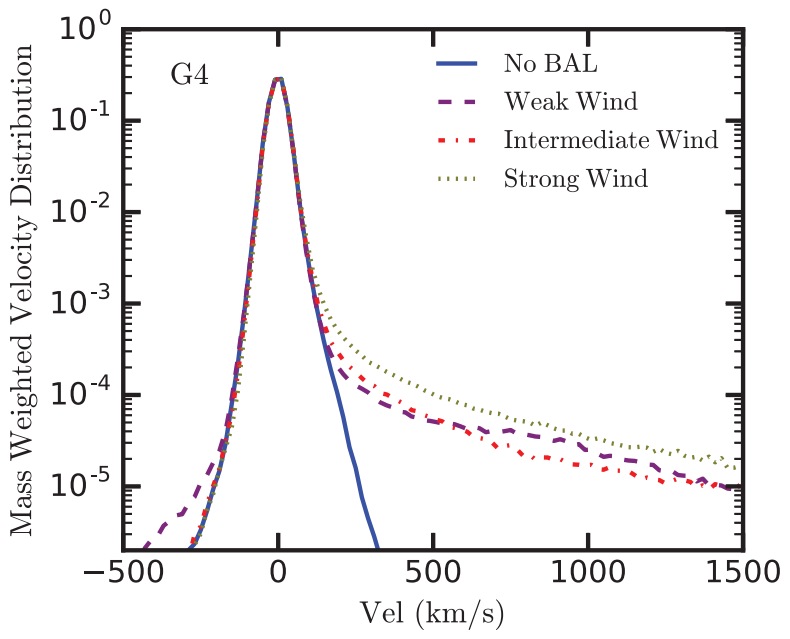

Figure 7. Distribution functions for the gas radial velocity for the $\mathrm{G} 4$ galaxy simulated with the three wind strengths and the no BAL simulation, as indicated in the legend. The distribution function is dominated by a nearly symmetric distribution about zero that reflects that most of the gas in each galaxy is in the disc-plane ISM, which is not immediately/strongly impacted by the BAL feedback.

In the absence of BAL feedback, the radial velocity distribution function is set by stellar feedback alone. The no BAL feedback simulations (solid blue lines in all panels) have radial velocity distributions that are reasonably well described by a zero-centred Gaussian distribution plus a lightly populated tail toward higher $\left(\sim 300-500 \mathrm{~km} \mathrm{~s}^{-1}\right)$ radial velocities. The zero-centred Gaussian - where the overwhelming majority of the gas resides - reflects the ISM gas velocity dispersion, driven by stellar feedback. The sparsely populated high-velocity tail reflects material in an outflow that is being driven by stellar feedback. We note that since both star formation and its associated feedback are somewhat bursty in these simulations, the amount of mass in the high-velocity outflow tail varies noticeably with time.

When BAL winds are introduced, the zero-centred Gaussian portion of the radial velocity distribution is not strongly impacted. As seen the previous section, the impact of the BAL feedback can be very pronounced within the central $\sim 1 \mathrm{kpc}$, but is less impactful on the more distant disc-plane ISM gas. The unmodified gas radial velocity distributions in this regime are consistent with the limited impact of the BAL feedback on the global ISM properties outside of the nuclear gas cavity. However, the high-velocity tail is strongly influenced as seen through a significant increase in the amount of high-velocity outflowing material that is present.

The high-velocity component of the radial velocity distribution is increasingly populated in the strong BAL wind simulations. Specifically, the high-velocity tail of the radial velocity distribution (i) contains more material and (ii) is shifted toward higher outflow velocities as the BAL direct injection velocity is increased. This is somewhat unsurprising since we are finding faster outflows in the case of faster wind injection speeds. We find that the radial velocity distribution function - even at large velocities - is dominated by ambient ISM material that was accelerated by the winds, not the BAL wind material itself. We note, however, that all of our simulations possess a high-velocity outflow, yet none of the simulations shown in Fig. 7 are rapidly quenching their host galaxy. In other words, the mere presence of a high-velocity outflow does not necessarily imply that galaxy-wide quenching will follow. This same qualitative picture holds true for the G4 simulation with increased black hole masses. Specifically, as we increase the AGN luminosity, the amount of material in the high velocity/momentum/energy tail of the gas distribution increases. For the most extreme case (G4_x10), the zerocentred Gaussian distribution is noticeably depressed owing to the significant impact of the BAL winds on the ambient ISM.

\subsection{Phase structure of outflows}

Using Fig. 7 as motivation, we define slow outflowing material as being all gas with outward radial velocities of $200>v_{\mathrm{r}}>500 \mathrm{~km} \mathrm{~s}^{-1}$ and rapidly outflowing material as being all gas with outward radial velocities of $v_{\mathrm{r}}>500 \mathrm{~km} \mathrm{~s}^{-1}$. The top row of Fig. 8 shows phase diagrams of the rapidly outflowing material from the G3 initial condition for the no, weak, intermediate, and strong BAL wind speed simulations, from left to right, respectively. The middle row of Fig. 8 shows the same information for slow outflowing material, while the bottom row displays the average radial velocity for all outflowing gas with $200 \mathrm{~km} \mathrm{~s}^{-1}>v_{\mathrm{r}}$ in this same space. Four coloured regions are indicated in the top row of Fig. 8 correspond to the regions of phase space where we broadly expect molecular (red), atomic (blue), warm/ionized (green), and hot (white) gas to reside. The dashed lines in each figure correspond to the theoretically expected post-shock gas temperature as described by equation (11). While we only show the phase diagrams for the G3 simulations, all of our conclusions are qualitatively identical when considering other initial condition galaxies.

We first note that most of the rapidly outflowing material in our simulations is hot (i.e. $T>10^{6} \mathrm{~K}$ ) and diffuse (i.e. $\rho<$ $0.1 \mathrm{~cm}^{-3}$ ) independent of the BAL input velocity. Within the hot gas distribution, there are two pockets of material that can be identified in the phase diagram that correspond to the post-shock BAL wind material and the entrained/accelerated ISM or off disc-plane material. The peaks corresponding to these gas populations are clearly visible in the weak and intermediate wind simulations, but also identifiable in the strong BAL wind simulation.

The majority of the mass of the rapid outflows is entrained/accelerated ambient gas that has a mass-weighted average temperature of just over $10^{6} \mathrm{~K}$ in all of the simulations - even as we vary the input BAL wind velocity over an order of magnitude. Most of this material was already warm (i.e. $\sim 10^{5}-10^{6} \mathrm{~K}$ ) off disc plane when the BAL central engine was turned on. It has not undergone a strong shock to reach these temperatures, but instead was accelerated through the off disc-plane pressure gradients found in Fig. 3.

The modest outflow velocity material shown in the middle row has some clear distinctions from the top row. In particular, while there is still a significant population of hot material (at $\gtrsim 10^{6} \mathrm{~K}$ ), there is also significant warm material $\left(10^{4}<T<10^{6} \mathrm{~K}\right)$. Tracking gas back in time indicates that the majority of this slow outflowing material was cool/cold disc-plane gas that has been accelerated without ever exceeding $\sim 10^{6} \mathrm{~K}$. Geometrically, while the majority of the fast outflowing material remains near the polar axis, the slow outflowing material tends to be further off-axis, above and below the disc plane.

Finally, examining the bottom panel of Fig. 8, we find that the majority of the entrained outflowing material has outflow velocities of just over $\sim 200 \mathrm{~km} \mathrm{~s}^{-1}$. Faster outflows are restricted to the hotter gas. For the intermediate and strong BAL wind simulations, we find there is a wider range of outflow velocities associated with the entrained material ranging from $\sim 500$ to $\sim 2000 \mathrm{~km} \mathrm{~s}^{-1}$.

The post-shock BAL wind material appears in the phase diagrams at $T \sim 10^{7.5}, \sim 10^{8.5}$, and $\sim 10^{9.5} \mathrm{~K}$, in the centre-left, centre-right, and right-hand panels, respectively. This gas population falls just below 

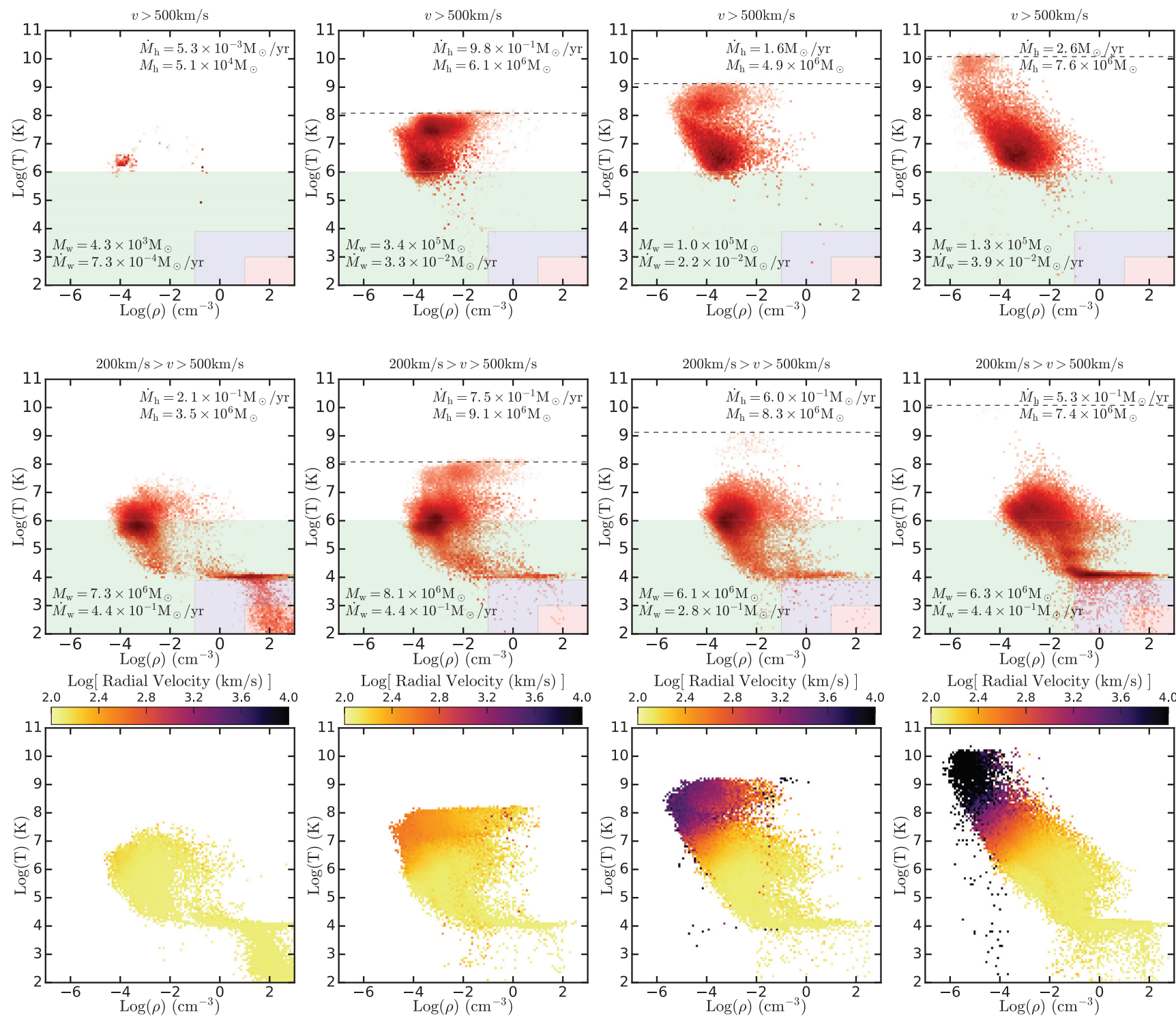

Figure 8. Temperature-density phase diagrams for the G3 galaxy showing the distribution of rapidly outflowing wind material (i.e. $v_{\mathrm{r}}>500 \mathrm{~km} \mathrm{~s}{ }^{-1}$; top row), modest speed outflowing material (i.e. $200<v_{\mathrm{r}}<500 \mathrm{~km} \mathrm{~s}^{-1}$; middle row), and the average radial velocity at a given $n$ and $T$ (bottom row). The four columns correspond to the no BAL simulation (left), weak winds (centre-left), intermediate winds (centre-right), and strong winds (right). The white, green, blue, and pink coloured background areas in the top and middle rows roughly indicate a divide between hot, warm/ionized, atomic, and molecular gas. The vertical dashed line (when present) identifies the expected post-shock gas temperature. Outflow rates for the hot and warm/ionized material are indicated within the three top plots. There is a dearth of cool and cold material in the simulated rapid outflows. The bulk of the rapidly outflowing material has temperatures $T>10^{6} \mathrm{~K}$ for all four simulations.

the horizontal dashed line in each panel, which corresponds to the temperature expected for the gas having passed through a shock with the BAL wind velocity (see equation 11). For the weak BAL wind case, the highest gas temperature agrees almost identically with the expected post-shock gas temperature. For the strong wind case, we find that there is some material with temperatures consistent with the expected post-shock gas temperature, but the majority of the hot post-shock gas is somewhat colder. The offset between the maximum gas temperature and the theoretically expected post-shock gas temperature is primarily driven by two effects: (i) adiabatic expansion of the post-shock heated gas, and (ii) actual shock velocities that are lower than the BAL wind velocity. Adiabatic expansion of the postshock gas appears to impact the fast wind simulations more severely because the post-shock gas simply expands more rapidly. The second effect is a result of considering the velocity of the ambient gas that the BAL wind material will run into. BAL wind material that is driven exactly into the disc mid-plane quickly encounters a static ISM with a negligible recession velocity (e.g. see Fig. 3) leading to rapid shock heating to the expected post-shock gas temperature. However, especially for the high BAL wind velocity simulations, the disc/cavity geometry is such that the majority of BAL wind material is launched at least slightly off the disc plane. The off disc-plane BAL wind material still shocks, but does so by running into either a diffusely populated CGM gas reservoir or previously launched BAL wind material, both of which can have significant recessional velocities (see Fig. 3). 


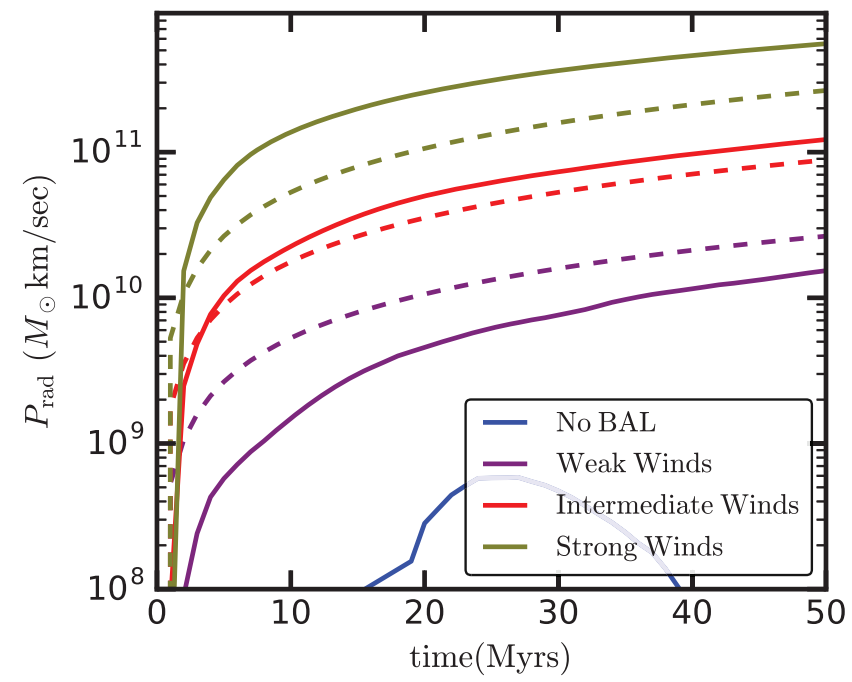

Figure 9. Radial momentum as a function of time for fast wind material (i.e. $v_{\mathrm{r}}>500 \mathrm{~km} \mathrm{~s}^{-1}$ ) in the $\mathrm{G} 3$ disc with no, weak, intermediate, and strong BAL feedback, as indicated in the legend. Solid lines indicate the instantaneously measured gas radial momentum. Dashed lines indicate the cumulative 'direct' momentum injection from the BAL wind. The outflowing material in the strong wind simulation has excess radial momentum beyond the direct injection value as a result of sweeping-up/accelerating significant amounts of ambient disc material.

\subsection{Total outflow momentum}

The final property of the outflows that we consider in this section is the total radial momentum of the outflows. The rate of BAL direct radial momentum injection for our simulations is straightforwardly calculated as $\dot{p}=\dot{M}_{\mathrm{BAL}} v_{\mathrm{BAL}}$. Since the accretion rates and wind velocities are both manually prescribed in this work, the direct momentum injection rate is a constant for each simulation. The solid lines in Fig. 9 show the measured rapidly outflowing (i.e. $v_{\mathrm{r}}>$ $500 \mathrm{~km} \mathrm{~s}^{-1}$ ) radial momentum that can be compared against the total direct injected radial momentum (dashed lines) as a function of time.

As is evident from Fig. 9, it is possible for the total rapid outflow radial momentum to be below, equal, or above the directly injected radial momentum from the BAL winds. In general, radial momentum is not a conserved quantity. Importantly for our simulations, the radial momentum of an outflow can be increased when overpressurized gas accelerates ambient material, or decreased when gas climbs out of a potential well. Our results indicate that the total radial momentum of the outflow depends on the strength of the BAL winds. Beyond the directly injected radial momentum, there is a boost in the total radial momentum of outflowing material that increases with the strength of the injected BAL winds. While the weak BAL wind simulations yield outflows with reduced total radial momentum, the strong BAL wind simulations show boosts to the total radial momentum of the outflowing material. While the weak BAL wind simulations fail to accelerate a significant fraction of the disc material to high radial velocities (see Fig. 7), the strong BAL wind simulations accelerate material both via shocks and strong pressure gradients. Further, we note that while all outflows in Fig. 9 are energy driven (in the sense that the hot wind bubbles do not cool), these outflows can have apparent momentum boost values above or below unity, depending on how the boost is defined. For example, the results in Fig. 9 include only gas moving at $>500 \mathrm{~km} \mathrm{~s}^{-1}$, so any momentum in swept up gas that has decelerated by momentum loading would be missed. While large radial momentum boosts are indicative of an energy-

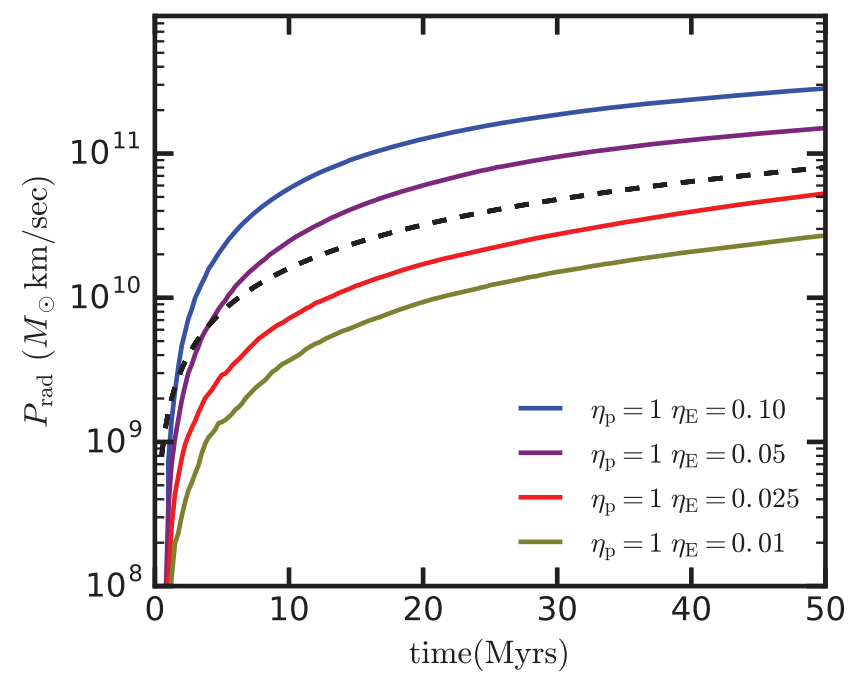

Figure 10. Radial momentum as a function of time for fast wind material (i.e. $v_{\mathrm{r}}>500 \mathrm{~km} \mathrm{~s}^{-1}$ ) in the $\mathrm{G} 3$ disc with varied direct energy injection values, as indicated in the legend. While all runs share the same 'direct momentum injection' rate (indicated with the black dashed line), the wind speed is varied such that fast winds carrying more energy than their slow counterparts. There is a clear trend where more energetic winds lead to larger momentum values in the outflows.

driven outflow, momentum boost factors of order unity (or even below unity) do not necessarily indicate a momentum-driven wind.

Interpreting the driving trend of increasing radial momentum boost with increasing wind strength shown in Fig. 9 is complicated by the fact that the weak, intermediate, and strong winds employed in this paper vary both the directly injected BAL wind energy and momentum. To disentangle the impact of modifying the direct BAL energy and momentum, we run four additional simulations of the G3 disc employing a constant direct momentum injection rate $\left(\eta_{p}=1\right)$, but varied energy injection rates $\left(\eta_{E}=0.1,0.05\right.$, 0.025 , and 0.0 ). Fig. 10 shows the total radial momentum measured for rapidly outflowing material as a function of time for these four additional simulations. We note that in Fig. 10 there is only one dashed line, which specifies the direct momentum injection for all four simulations. There is a clear differentiation between the total radial momentum of the outflows where the more energetic winds give rise to larger measured outflow radial momentum. We note here, but discuss further in Section 5, that numbers quoted for the total radial momentum boost are likely to change in simulations that include more substantial CGM where the wind venting process is further constrained.

\section{DISCUSSION}

In this paper, we have presented three-dimensional galaxy-scale simulations including both stellar feedback and AGN feedback using a novel BAL winds feedback scheme. Our simulations develop a central hot gas cavity that in many ways is in agreement with previously explored analytic arguments (King \& Pounds 2003; Faucher-Giguère \& Quataert 2012) and numerical simulations (Costa et al. 2014; Richings \& Faucher-Giguère 2018a). Specifically, we find a very high temperature/pressure gas cavity that expands along the path of least resistance. For the idealized and isolated discs considered in this paper, the BAL wind feedback drives low-density outflow cavities that extend primarily in the polar directions. 


\subsection{Phase structure of outflows}

We have considered the properties of the outflows including an examination of the phase structure of the driven outflows. We found that the rapid outflows (i.e. $v_{\mathrm{r}}>500 \mathrm{~km} \mathrm{~s}^{-1}$ ) in our simulations are comprised almost exclusively of hot gas (i.e. $>10^{6} \mathrm{~K}$ ), with a larger presence of warm/ionized gas for modest outflow velocities (i.e. $200<v_{\mathrm{r}}<500 \mathrm{~km} \mathrm{~s}^{-1}$ ). Both the hot and warm/ionized fast outflow mass budgets are dominated by shocked ambient ISM material. While hot/warm outflows are plentiful in our simulations, our simulations do not produce significant fast atomic and molecular outflows that are observed around some quasar systems (e.g. Rupke \& Veilleux 2011; Sturm et al. 2011; Cicone et al. 2014; Kakkad et al. 2017).

The lack of cold gas in our simulated outflows raises questions about the ability of fast BAL winds to drive cool and cold gas outflows found in quasar hosts. However, we stress that the properties of the outflows are strongly coupled to the gas geometry about the central black hole. In the simulations considered in this paper, which are limited to idealized and isolated galaxies with reasonably thin ISM gas discs, the post-shock gas rapidly vents through low-density polar cavities without strongly interacting with the cool and cold ISM gas. Specifically, once the central gas bubble becomes roughly equal to the scale height of the ISM, the hot gas will break out in the polar directions and provide a preferred venting channel. It is therefore somewhat unsurprising that our simulation outflows are dominated by very hot gas.

Our results may qualitatively change if the gas geometry around the central black hole is substantially modified. For example, galaxy mergers have the ability to rapidly funnel large amounts of ISM gas into galactic nuclei, which can result in elevated SFRs and elevated black hole accretion rates. At the same time, the rapid influx of gas changes the central gas geometry, which could be better approximated by a quasi-isotropic ambient medium than the disc geometry explored in this paper. Richings \& FaucherGiguère (2018a) studied the coupling of BAL wind mechanisms to uniform density medium and found large, cold molecular outflows rates resulted for luminous quasar parameters. It is also possible that more persistent modifications to the ISM gas geometry may also play a role. For example, high-redshift galaxies are observed to be more compact with higher SFRs, higher gaseous velocity dispersions, and thicker gas disc scale heights. Increased gas disc scale heights increase the opening angle of the ISM disc as seen by the black hole $(h / r)$, likely increasing the coupling efficiency of AGN wind even in galaxies where a significant merger has not recently occurred.

Finally, we should note that even the idealized discs considered here have elements of incompleteness or at least uncertainty in their construction. In particular, the isolated galaxies explored in this paper are not initialized with a massive gaseous hot halo (e.g. as in Moster, Macciò \& Somerville 2014). After their 1 Gyr relaxation period, the galaxy models do contain significant mass above the disc plane. However, while the total hot halo gas mass for Milky Way (MW) mass galaxies is not well known, the total mass of the hot halo gas used in our simulations is likely below even conservative estimates. This can change in detail some of the properties of the hot wind bubble as it expands in the polar directions. In particular, the extra material along the venting channels may give rise to larger mass loadings for the outflows, reduced wind speeds, and possibly additional phase structure in the outflowing gas.

Aside from the two varied galaxy models considered in this paper, we have not carried out an extended study of the impact of local gas geometry on the coupling efficiency of AGN feedback. We reserve a comparison of the coupling efficiency and outflow properties of our BAL winds in galaxy mergers and a more systematic exploration of the impact on host galaxy gas distribution for a future paper.

\subsection{Implications for AGN-galaxy coevolution?}

Our simulations are intended to study one possibly important AGN feedback channel in idealized galaxies where we can begin to address the mechanisms that allow AGN feedback to couple (or not) galaxy wide. In particular, the method for modelling BAL winds used in this paper by launching fast material near the accreting black hole is constructed to capture the essential physics of one of the main feedback mechanisms associated with quasars. Even with our limited sets of simulations, we find there are regimes where AGN feedback drives massive outflows and quenches star formation, and regimes where AGN feedback has a limited impact on the host galaxy.

Throughout all of our simulations, we find that the fast winds driven by an AGN couple inefficiently to thin gas discs. As a result, some of our isolated disc galaxies are impacted to a very limited extent since most of the energetic gas is vented in the polar directions. This result of AGN having limited impact on their host galaxies can hold up to intermediate-luminosity $\mathrm{AGN}\left(L_{\mathrm{AGN}} \lesssim 10^{46} \mathrm{erg} \mathrm{s}^{-1}\right.$ for the relatively massive galaxies that are the focus of this study). This result may explain why observed weak- and intermediate-luminosity AGN that are found in disc-dominated (e.g. Gabor et al. 2009; Schawinski et al. 2011; Kocevski et al. 2012) star-forming (Rosario et al. 2013) galaxies often have limited signs of ongoing quenching. Even though the AGN themselves may be sufficiently energetic to have a strong impact on their host galaxy's star formation, the feedback from these central engines will not necessarily couple efficiently to the dense ISM, and therefore will have a limited impact on SFRs. Similar conclusions were found by Gabor \& Bournaud (2014) and Roos et al. (2015) who found that AGN feedback had only a mild impact on the host galaxy in the short term owing to the tendency of feedback-driven material to exit the galaxy along the paths of least resistance.

However, our simulations also indicate there are regimes where AGN feedback can have a significant impact on the host galaxy in spite of the inefficient coupling. In particular, we find that quenching can be achieved in disc galaxies by increasing the AGN luminosity, without modifying the AGN feedback mechanism, disc properties, or coupling efficiency. In particular, our simulated galaxies with higher AGN luminosities (i.e. $L_{\mathrm{AGN}} \sim 10^{47} \mathrm{erg} \mathrm{s}^{-1}$ ) showed significant central gas blowouts and quenched rapidly. These systems included Eddington accretion rates and black hole masses at the upper end but within the scatter - of the $M_{\mathrm{BH}}-M_{\text {bulge }}$ relation (see Kormendy \& Ho 2013).

Given that a majority of observed AGN do not appear to be impacting their host galaxies, it has been argued that AGN may not be able to couple their energy within galaxies sufficiently efficiently to act as a primary driver of quenching. Our simulations are able to reproduce low and intermediate AGN feedback models that have a limited impact on their host galaxies. Despite similarities in the employed methods, our conclusion of inefficient AGN feedback coupling stands in contrast to the conclusions on some earlier studies (e.g. Choi et al. 2012). Instead, our simulations suggest that AGN feedback impact can be very important at the higher AGN luminosities, if the feedback can be sustained for $\sim 20-30$ Myr. Further, a physical reason for the limited AGN feedback impact in our simulations is the disc-like geometry, which was set by our choice 
of initial conditions and allowed for energy to be vented in the polar directions. We expect that higher coupling efficiencies in systems with non-disc-like gas morphology including, e.g. galaxy mergers or high-redshift, thick, highly turbulent discs. In those cases, the AGN feedback efficiency will likely be higher owing to the lack of a clear polar venting channel (though, see Costa et al. 2014; Curtis \& Sijacki 2016, for an exploration of modified gas geometries that did not show significant feedback boosting efficiency).

\subsection{Numerical modelling considerations}

Implementing a physically faithful scheme to capture the multiscale physical processes involved in AGN feedback is notoriously challenging. To this point we have neglected a discussion on numerical considerations, including numerical convergence and an exploration of varied AGN feedback implementation choices, both of which have a moderate impact on our results. In the case of assessing numerical convergence, two main issues arise. First, our simulations employ the FIRE model, and the isolated discs themselves have modest changes in their properties as a function of resolution [e.g. the giant molecular clouds (GMC) mass function, the SFR, and the nuclear gas density]. While the resolution dependence of this model has been addressed in the literature, even modest changes to the local gas geometry around the supermassive black hole can have a significant impact on e.g. the efficiency of wind coupling and the central cavity structure. Put another way, while we do find variability in the cavity size evolution and level of SFR suppression that occurs when we change our simulated galaxies resolution, we also find variability in those same quantities when we shift the starting time for our initial conditions owing to detailed changes in the detailed gas properties of the disc. Thus, presenting a clean assessment of the numerical convergence of the AGN feedback model that is at the heart of this paper is uniquely challenging.

One particular issue that could directly depend on numerical resolution is the ability to fully resolve the wind shock structure. Depending on the scale where the wind shock is resolved, part of the energy conserving expansion phase can be missed. Specifically, failing to resolve scales where the reverse shock first thermalizes the wind material would result in an underrepresentation of the energy conserving expansion phase and therefore an underprediction for the momentum boost of the AGN-driven outflow. We do find factor of $\sim 2$ changes that occur in our results (specifically the SFRs and cavity sizes) when the resolution is changed. However, the sense of those resolution-dependent changes is not a consistent function of resolution change, and also vary with the employed disc. This suggests that while it is possible that the simulations in this paper are partially underresolving early energy conserving expansion, our results are likely not dominated by resolution effects.

We note that we performed tests employing a similar AGN feedback scheme but in which the BAL wind particles were spawned directly with the post-shock wind properties (i.e. with post-shock temperature up to $T \sim 10^{10} \mathrm{~K}$ ). That scheme effectively removes the concerns that shock heating owing to inner, reverse shock is not properly captured. Similar to our variable resolution tests, those simulations yielded qualitatively indistinguishable results, but had quantitative variations in the predicted cavity sizes, degree of SFR suppression, or momentum boost factors that changed by factors of a few. We thus encourage caution with overinterpreting the quantitative results presented in this paper but stress that our qualitative results are robust with respect to resolution and numerical implementation.

\subsection{AGN modelling completeness}

Our simulations do not attempt to include a number of other possibly associated AGN feedback effects including e.g. radiation pressure, photoionization, or jets. While strong jets are not present in most AGN, radiation pressure and photoionization will in general be both present in luminous AGN that drive accretion disc winds.

Finally, it is worth emphasizing that we have employed fixed accretion rates in our simulations, without allowing the black holes to 'self-regulate' their growth. This was a deliberate choice to simplify interpretation in our initial exploration of our new BAL wind feedback scheme. However, this can potentially lead to overestimates about the size of the central cavity - especially in the strong feedback simulations. The intermediate and strong BAL wind simulations regularly showed an ability to carve out a central low-density gas cavity. In reality, we would expect low central gas densities to lead to a reduction in the black hole accretion rates and feedback strengths. This effect is not present in our simulations. Instead, by holding the black hole accretion rates fixed, we can interpret our results as upper limits on the possible impact of our BAL wind feedback scheme on the host galaxy. Including self-consistent accretion rates would possibly reduce the central cavity sizes that are formed in the strong feedback simulations.

\section{CONCLUSIONS}

In this paper, we used numerical simulations to study the evolution of an energetic, kinetic wind that is launched from a central, accreting supermassive black hole. We ran idealized/isolated galaxy simulations using the FIRE feedback model in conjunction with a newly developed BAL wind feedback method (Section 2). We examined the impact of the BAL winds on the host galaxy as they expand out into the porous ISM. We found that the BAL winds from luminous AGN can have a significant impact on their host galaxy gas profiles and SFRs (Section 3). However, we also found that lower luminosity AGN had a limited impact on their host galaxies properties owing to the limited spatial extent to which the winds penetrated into the ISM disc (Section 3). We further found that the low-density polar directions provided escape channels for the energetic BAL wind material to vent. We analysed the properties of the outflows and found that while our simulations reproduce the fast outflow velocities seen in some quasar hosts, the overwhelming majority of our simulated outflow material would be hot and diffuse and therefore difficult to observe (Section 4). However, we did find that the outflows in the energetic BAL wind simulations undergo significant radial momentum boosting during an energy conserving expansion phase.

Although the energetic BAL winds couple relatively inefficiently to thin-disc host galaxies, we emphasize that AGN wind feedback may well be very important in high-luminosity AGN systems, or in systems with more complicated gas geometries. In particular, the coupling efficiency of BAL winds to the broader galactic scale ISM depends sensitively on the distribution of gas around the central black hole. This study was strictly limited to isolated, thin galactic disc geometries that leave natural venting channels in the polar directions that the energetic winds can vent through. We expect that our conclusions may qualitatively change when we consider either significantly thicker gas discs or merging galaxies. In both cases, the ease with which the energetic winds can escape the galaxy will be reduced. This may lead to a picture where the efficiency of AGN feedback is tied not just to the black hole accretion rate, but also to the geometry of the gas around the black hole. 


\section{ACKNOWLEDGEMENTS}

We thank Alexander Richings for useful discussions. PT was supported by NSF through grant AST-1909933 and NASA ATP Grant 19-ATP19-0031. Support for PFH was provided by an Alfred P. Sloan Research Fellowship, NASA ATP Grant NNX14AH35G, NSF Collaborative Research Grant \#1411920, and CAREER grant \#1455342. C-AF-G was supported by NSF through grants AST1517491, AST-1715216, and CAREER award AST-1652522, by NASA through grant 17-ATP17-0067, by CXO through grant TM718007, and by a Cottrell Scholar Award from the Research Corporation for Science Advancement. DK was supported by NSF grant AST-1715101 and the Cottrell Scholar Award from the Research Corporation for Science Advancement. This work was supported in part by a Simons Investigator Award from the Simons Foundation (EQ) and by NSF grant AST-1715070. RF acknowledges financial support from the Swiss National Science Foundation (grant no. 157591). This research was undertaken, in part, thanks to funding from the Canada Research Chairs program. The simulations reported in this paper were run and processed on the 'Quest' computer cluster at Northwestern University, the Caltech compute cluster 'Zwicky' (NSF MRI award \#PHY-0960291), and allocation TGAST130039, TG-AST150059, and TG-AST140076 granted by the Extreme Science and Engineering Discovery Environment (XSEDE) supported by the NSF (Towns et al. 2014).

\section{DATA AVAILABILITY}

The data underlying this paper will be shared on reasonable request to the corresponding author.

\section{REFERENCES}

Anglés-Alcázar D., Özel F., Davé R., 2013, ApJ, 770, 5

Anglés-Alcázar D., Özel F., Davé R., Katz N., Kollmeier J. A., Oppenheimer B. D., 2015, ApJ, 800, 127

Anglés-Alcázar D., Davé R., Faucher-Giguère C.-A., Özel F., Hopkins P. F., 2017a, MNRAS, 464, 2840

Anglés-Alcázar D., Faucher-Giguère C.-A., Kereš D., Hopkins P. F., Quataert E., Murray N., 2017b, MNRAS, 470, 4698

Anglés-Alcázar D., Faucher-Giguère C.-A., Quataert E., Hopkins P. F., Feldmann R., Torrey P., Wetzel A., Kereš D., 2017c, MNRAS, 472, L109

Cappi M. et al., 2009, A\&A, 504, 401

Carniani S. et al., 2015, A\&A, 580, A102

Carniani S. et al., 2016, A\&A, 591, A28

Chan T. K., Kereš D., Oñorbe J., Hopkins P. F., Muratov A. L., FaucherGiguère C.-A., Quataert E., 2015, MNRAS, 454, 2981

Choi E., Ostriker J. P., Naab T., Johansson P. H., 2012, ApJ, 754, 125

Choi E., Somerville R. S., Ostriker J. P., Naab T., Hirschmann M., 2018, ApJ, 866, 91

Cicone C. et al., 2014, A\&A, 562, A21

Ciotti L., Ostriker J. P., 1997, ApJ, 487, L105

Costa T., Sijacki D., Haehnelt M. G., 2014, MNRAS, 444, 2355

Costa T., Rosdahl J., Sijacki D., Haehnelt M. G., 2018, MNRAS, 479, 2079

Curtis M., Sijacki D., 2016, MNRAS, 463, 63

Di Matteo T., Springel V., Hernquist L., 2005, Nature, 433, 604

Dunn J. P. et al., 2010, ApJ, 709, 611

El-Badry K., Wetzel A., Geha M., Hopkins P. F., Kereš D., Chan T. K., Faucher-Giguère C.-A., 2016, ApJ, 820, 131

Faucher-Giguère C.-A., Quataert E., 2012, MNRAS, 425, 605

Faucher-Giguère C.-A., Quataert E., Murray N., 2012, MNRAS, 420, 1347

Faucher-Giguère C.-A., Hopkins P. F., Kereš D., Muratov A. L., Quataert E., Murray N., 2015, MNRAS, 449, 987

Faucher-Giguère C.-A., Feldmann R., Quataert E., Kereš D., Hopkins P. F., Murray N., 2016, MNRAS, 461, L32
Ferrarese L., Merritt D., 2000, ApJ, 539, L9

Fiore F. et al., 2017, A\&A, 601, A143

Fluetsch A. et al., 2019, MNRAS, 483, 4586

Gabor J. M., Bournaud F., 2014, MNRAS, 441, 1615

Gabor J. M. et al., 2009, ApJ, 691, 705

Gebhardt K. et al., 2000, ApJ, 539, L13

Greene J. E., Pooley D., Zakamska N. L., Comerford J. M., Sun A.-L., 2014, ApJ, 788, 54

Hafen Z. et al., 2017, MNRAS, 469, 2292

Harrison C. M., Alexander D. M., Mullaney J. R., Swinbank A. M., 2014, MNRAS, 441, 3306

Heckman T. M., Best P. N., 2014, ARA\&A, 52, 589

Hernquist L., 1990, ApJ, 356, 359

Ho L. C., 2008, ARA\&A, 46, 475

Hopkins P. F., 2015, MNRAS, 450, 53

Hopkins P. F., Hernquist L., 2006, ApJS, 166, 1

Hopkins P. F., Kereš D., Oñorbe J., Faucher-Giguère C.-A., Quataert E., Murray N., Bullock J. S., 2014, MNRAS, 445, 581

Hopkins P. F. et al., 2018a, MNRAS, 477, 1578

Hopkins P. F. et al., 2018b, MNRAS, 480, 800

Hopkins P. F., Grudic M. Y., Wetzel A. R., Keres D., Faucher-Giguère C.-A., Ma X., Murray N., Butcher N., 2020, MNRAS, 491, 3702

Jahnke K., Macciò A. V., 2011, ApJ, 734, 92

Jiang Y.-F., Stone J. M., Davis S. W., 2014, ApJ, 796, 106

Kakkad D. et al., 2017, MNRAS, 468, 4205

Kim C.-G., Ostriker E. C., Kim W.-T., 2013, ApJ, 776, 1

King A., 2003, ApJ, 596, L27

King A. R., Pounds K. A., 2003, MNRAS, 345, 657

Kocevski D. D. et al., 2012, ApJ, 744, 148

Kormendy J., Ho L. C., 2013, ARA\&A, 51, 511

Liu G., Zakamska N. L., Greene J. E., Nesvadba N. P. H., Liu X., 2013, MNRAS, 436, 2576

Ma X., Hopkins P. F., Faucher-Giguère C.-A., Zolman N., Muratov A. L., Kereš D., Quataert E., 2016, MNRAS, 456, 2140

Ma X., Hopkins P. F., Feldmann R., Torrey P., Faucher-Giguère C.-A., Kereš D., 2017, MNRAS, 466, 4780

Maiolino R. et al., 2012, MNRAS, 425, L66

Moe M., Arav N., Bautista M. A., Korista K. T., 2009, ApJ, 706, 525

Moster B. P., Macciò A. V., Somerville R. S., 2014, MNRAS, 437, 1027

Muratov A. L., Kereš D., Faucher-Giguère C.-A., Hopkins P. F., Quataert E., Murray N., 2015, MNRAS, 454, 2691

Muratov A. L. et al., 2017, MNRAS, 468, 4170

Murray N., Quataert E., Thompson T. A., 2005, ApJ, 618, 569

Nardini E. et al., 2015, Science, 347, 860

Nesvadba N. P. H., Lehnert M. D., Eisenhauer F., Gilbert A., Tecza M., Abuter R., 2006, ApJ, 650, 693

Nesvadba N. P. H., Lehnert M. D., De Breuck C., Gilbert A. M., van Breugel W., 2008, A\&A, 491, 407

Nomura M., Ohsuga K., 2017, MNRAS, 465, 2873

Nomura M., Ohsuga K., Wada K., Susa H., Misawa T., 2013, PASJ, 65, 40

Oñorbe J., Boylan-Kolchin M., Bullock J. S., Hopkins P. F., Kereš D., Faucher-Giguère C.-A., Quataert E., Murray N., 2015, MNRAS, 454, 2092

Ostriker J. P., Choi E., Ciotti L., Novak G. S., Proga D., 2010, ApJ, 722, 642

Peng C. Y., 2007, ApJ, 671, 1098

Proga D., Stone J. M., Kallman T. R., 2000, ApJ, 543, 686

Richings A. J., Faucher-Giguère C.-A., 2018a, MNRAS, 474, 3673

Richings A. J., Faucher-Giguère C.-A., 2018b, MNRAS, 478, 3100

Risaliti G., Elvis M., 2010, A\&A, 516, A89

Roos O., Juneau S., Bournaud F., Gabor J. M., 2015, ApJ, 800, 19

Rosario D. J. et al., 2013, ApJ, 771, 63

Rupke D. S. N., Veilleux S., 2011, ApJ, 729, L27

Rupke D. S. N., Veilleux S., 2013, ApJ, 768, 75

Sagdowski A., Narayan R., Penna R., Zhu Y., 2013, MNRAS, 436, 3856

Sazonov S. Y., Ostriker J. P., Sunyaev R. A., 2004, MNRAS, 347, 144

Schawinski K., Treister E., Urry C. M., Cardamone C. N., Simmons B., Yi S. K., 2011, ApJ, 727, L31 
Sijacki D., Springel V., Di Matteo T., Hernquist L., 2007, MNRAS, 380, 877 Sijacki D., Vogelsberger M., Genel S., Springel V., Torrey P., Snyder G. F., Nelson D., Hernquist L., 2015, MNRAS, 452, 575

Silk J., Rees M. J., 1998, A\&A, 331, L1

Springel V., Di Matteo T., Hernquist L., 2005, MNRAS, 361, 776

Stern J., Faucher-Giguère C.-A., Zakamska N. L., Hennawi J. F., 2016, ApJ, 819,130

Sturm E. et al., 2011, ApJ, 733, L16

Teyssier R., Moore B., Martizzi D., Dubois Y., Mayer L., 2011, MNRAS, 414, 195

Tombesi F., Cappi M., Reeves J. N., Palumbo G. G. C., Yaqoob T., Braito V., Dadina M., 2010, A\&A, 521, A57

Tombesi F., Meléndez M., Veilleux S., Reeves J. N., González-Alfonso E., Reynolds C. S., 2015, Nature, 519, 436

Torrey P., Hopkins P. F., Faucher-Giguère C.-A., Vogelsberger M., Quataert E., Kereš D., Murray N., 2017, MNRAS, 467, 2301

Towns J. et al., 2014, Comput. Sci. Eng., 16, 62
Tremmel M., Karcher M., Governato F., Volonteri M., Quinn T. R., Pontzen A., Anderson L., Bellovary J., 2017, MNRAS, 470, 1121

Veilleux S., Teng S. H., Rupke D. S. N., Maiolino R., Sturm E., 2014, ApJ, 790,116

Wagner A. Y., Bicknell G. V., Umemura M., 2012, ApJ, 757, 136

Wagner A. Y., Umemura M., Bicknell G. V., 2013, ApJ, 763, L18

Weymann R. J., Carswell R. F., Smith M. G., 1981, ARA\&A, 19, 41

Williams R. J., Maiolino R., Krongold Y., Carniani S., Cresci G., Mannucci F., Marconi A., 2017, MNRAS, 467, 3399

Wyithe J. S. B., Loeb A., 2003, ApJ, 595, 614

Wylezalek D., Zakamska N. L., 2016, MNRAS, 461, 3724

Zakamska N. L. et al., 2016, MNRAS, 459, 3144

Zubovas K., 2018, MNRAS, 479, 3189

Zubovas K., Bourne M. A., 2017, MNRAS, 468, 4956

This paper has been typeset from a $\mathrm{T}_{\mathrm{E}} \mathrm{X} / \mathrm{LT} \mathrm{T} \mathrm{X}$ file prepared by the author. 